\title{
DERIVATIONS OF VON NEUMANN ALGEBRAS INTO THE COMPACT IDEAL SPACE OF A SEMIFINITE ALGEBRA
}

\author{
SORIN POPA AND FLORIN RĂDULESCU
}

1. Introduction and statement of results. Let $M$ be a semifinite von Neumann algebra and let $\mathscr{J}(M)$ be the norm closed two-sided ideal generated by the finite projections of $M$. Let $N \subseteq M$ be a subalgebra of $M$. A derivation of $N$ into $\mathscr{J}(M)$ is a linear application $\delta: N \mapsto \mathscr{J}(M)$ satisfying $\delta(x y)=\delta(x) y+x \delta(y)$ for $x, y \in N$. For instance, if $K \in \mathscr{J}(M)$, then the derivation $\delta(x)=(\operatorname{ad} K)(x)$ $=K x-x K$ is of this type. Such derivations implemented by elements in $\mathscr{J}(M)$ are called inner. There are many examples of derivations of *-subalgebras $N \subseteq M$ into the ideal $\mathscr{J}(M)$ which are not inner. A typical such example is as follows: Take $M=\mathscr{B}\left(\mathrm{L}^{2}(\mathrm{~T}, \mu)\right)$, where $\mu$ is the Lebesgue measure on the torus $\mathrm{T}$, let $N=\mathrm{C}(\mathbb{T})$ act on $\mathrm{L}^{2}(\mathrm{~T}, \mu)$ by left multiplication, and define $\delta(x)=$ $\left(\operatorname{ad} \mathrm{P}_{\mathrm{H}^{2}}\right)(x)$, where $\mathrm{P}_{\mathrm{H}^{2}}$ is the projection onto the Hardy subspace $\mathrm{H}^{2}(\mathbb{T}, \mu)$ ([1], [11]). Then it is easy to see that $\delta(x) \in \mathscr{K}(\mathscr{H})=\mathscr{J}(\mathscr{B}(\mathscr{H}))$ for $x \in \mathrm{C}(\mathbb{T})$ and that $\delta$ is not implemented by a compact operator.

We will, however, show in this paper that if $N$ is self-adjoint and $w$-closed in $M$, then, except for certain situations, all derivations of $N$ into $\mathscr{J}(M)$ are inner. Moreover, for the most typical excepted case we'll construct a counterexample.

This derivation problem was initiated in the case $M=\mathscr{B}(\mathscr{H})$ and $\mathscr{J}(M)=$ $\mathscr{K}(\mathscr{H})$ by Johnson and Parrott in a paper of the early '70s ([3]). In that paper Johnson and Parrott wanted to characterize the commutant modulo the ideal of compact operators $\mathscr{K}(\mathscr{H}) \subseteq \mathscr{B}(\mathscr{H})$ for a von Neumann algebra $N \subseteq \mathscr{B}(\mathscr{H})$. They noted that in order to identify it with the compact perturbations of the commutant of $N$ in $\mathscr{B}(\mathscr{H})$, it suffices to show that any derivation $\delta: N \mapsto \mathscr{K}(\mathscr{H})$ is inner. They proved that this is indeed the case if $N$ has no certain type $\mathrm{II}_{1}$ factors as direct summands. To do this they first solved the case when $N$ is abelian, the other cases being rather easy consequences of it. The general type $\mathrm{II}_{1}$ case was proved recently in [7] by different techniques and using more of the ergodic theory of the type $\mathrm{II}_{1}$ factors.

In [4] this derivation problem is studied in the more general setting when $\mathscr{B}(\mathscr{H})$ is replaced by a semifinite von Neumann algebra, $\mathscr{K}(\mathscr{H})$ by the ideal $\mathscr{J}(M)$, and the center of $N$ is assumed to contain the center of $M$. Under this hypothesis it is proved that if $N$ is either an abelian or a properly infinite von Neumann algebra, then any derivation of $N$ into $\mathscr{J}(M)$ is inner.

Received March 31, 1986. Revision received September 10, 1987. 
To state our results in precise terms, let us first recall that any von Neumann algebra $N$ can be decomposed into a direct sum $N=N_{0} \oplus N_{1}$ with $N_{0}$ a finite type I von Neumann algebra and $N_{1}$ a von Neumann algebra that has no finite type I summands. We will then say that $N_{0}$, as a subalgebra in $M$, is locally compatible with the center of $M, \mathscr{Z}(M)$, if there exists a partition of the unity $\left\{p_{i}\right\}_{i \in I}$ in the center of $N_{0}, \mathscr{Z}\left(N_{0}\right)$, so that for each $i$ we have either $\mathscr{Z}\left(N_{0}\right) p_{i} \subseteq \mathscr{Z}(M) p_{i}$ or $\mathscr{Z}(M) p_{i} \subseteq \mathscr{Z}\left(N_{0}\right) p_{i}$.

1.1. TheOREM. Let $M$ be a semifinite von Neumann algebra and $\mathscr{J}(M)$ its compact ideal space. Let $N \subset M$ be a weakly closed ${ }^{*}$-subalgebra of $M$ and suppose the finite type I summand of $N$ is locally compatible with the center of $M$ (in the sense described above). Then any derivation of $N$ into $\mathscr{J}(M)$ is inner. Moreover, if $\delta: \quad N \rightarrow \mathscr{J}(M)$, then there exists $K \in \mathscr{J}(M),\|K\| \leqslant 2\|\delta\|$ with $\delta=\operatorname{ad} K$. In particular, the commutant modulo $\mathscr{J}(M)$ of $N$ in $M$ equals $N^{\prime} \cap M+\mathscr{J}(M)$.

Thus, Theorem 1.1 solves in the affirmative the derivation problem if $N$ is of type $\mathrm{II}_{1}$ or properly infinite. It also gives an affirmative answer to the remaining case when $N$ is finite of type I (e.g., when $N$ is the tensor product of a matrix algebra with an abelian algebra) under an additional assumption of local compatibility between the centers of $N$ and $M$. The typical situation when this condition is not fulfilled is when $N$ is abelian and diffuse (i.e., without atoms), $\mathscr{Z}(M)$ is also diffuse, and $N$ and $\mathscr{Z}(M)$ are independent von Neumann algebras, namely, $N$ and $\mathscr{Z}(M)$ generate the von Neumann algebra $N \bar{\otimes} \mathscr{Z}(M)$ with $N, \mathscr{Z}(M)$ sitting inside it as $N \otimes 1$ and $1 \otimes \mathscr{Z}(M)$.

The second theorem that we will prove in this paper deals with the most simple such case, left open by Theorem 1.1 , namely, when $M=\mathrm{L}^{\infty}([0,1], \lambda) \bar{\otimes}$ $\mathscr{B}\left(\mathrm{L}^{2}(\mathbb{T}, \mu)\right)$ and $N=1 \otimes \mathrm{L}^{\infty}(\mathbb{T}, \mu)$. In this case we will construct a counterexample, showing the existence of a derivation of $N$ into $\mathscr{J}(M)$ not implemented by an element in $\mathscr{J}(M)$. This is somehow unexpected and is probably the first nonvanishing 1-cohomological result in von Neumann algebras. It practically shows that the one-parameter version of Johnson and Parrott's original result may fail to be true. In order to have an alternative, more intuitive interpretation of the next theorem, the reader should notice that we may identify $M=$ $\mathrm{L}^{\infty}([0,1], \lambda) \bar{\otimes} \mathscr{B}\left(\mathrm{L}^{2}(\mathbb{T}, \mu)\right)$ with $\mathrm{L}^{\infty}\left([0,1], \mathscr{B}\left(\mathrm{L}^{2}(\mathbb{T}, \mu)\right)\right), \mathscr{J}(M)$ with $\mathrm{L}^{\infty}\left([0,1], \mathscr{K}\left(\mathrm{L}^{2}(\mathbb{T}, \mu)\right)\right)$, and $N=1 \otimes \mathrm{L}^{\infty}(\mathbb{T}, \mu)$ with the set of constant $\left.\mathrm{L}^{\infty}(\mathbb{T}, \mu)\right)$ valued functions on the interval $[0,1]$.

1.2. TheOREM. Let $M=L^{\infty}([0,1], \lambda) \bar{\otimes} \mathscr{B}\left(L^{2}(\mathbb{T}, \mu)\right), N=1 \otimes L^{\infty}(\mathbb{T}, \mu) \subset$ $M$. There exists an operator $T \in M$ which commutes modulo $\mathscr{J}(M)$ with all the elements in $N$ but which is not a compact (i.e., $\mathscr{J}(M))$ perturbation of an element commuting with $M$. In particular, there exists a derivation $\delta(=\operatorname{ad} T)$ from $N$ into $\mathscr{J}(M)$ which is not inner, i.e., not implemented by an element in $\mathscr{J}(M)$.

The paper is organized as follows: In sections 2-7 we prove Theorem 1.1 and in section 8 we prove Theorem 1.2. We will now present some of the ideas behind the proof of Theorem 1.1. 
A key idea of our proof is to work with a new norm on the algebra $M$, denoted ||| |||, which in our problem turns out to be the right correspondent of the uniform norm on $\mathscr{B}(\mathscr{H})$. This norm has two main features: it helps one deal with the center of $M$ when diffuse and with the continuous dimension of projections when $M$ is of type $\mathrm{II}_{\infty}$. The definition and main properties of the norm |||| $\mid$ are discussed in section 2.

We then prove Theorem 1.1 in the case $N$ is atomic and abelian. In the proof we define the operator implementing $\delta$ as $\sum_{i} \delta\left(e_{i}\right) e_{i}$, where $e_{i}$ are the atoms of $N$ and the series is strongly convergent, and we use an adaptation of a trick in [3] to show that $\sum_{i} \delta\left(e_{i}\right) e_{i} \in \mathscr{J}(M)$.

By the atomic abelian case and by the same argument as in 4.1 [7] (for $M=\mathscr{B}(\mathscr{H})$ ) we prove a continuity result, namely, that if $N$ is finite and countably decomposable, then $\delta$ is continuous from the unit ball of $N$ with the strong operator topology into $\mathscr{J}(M)$ with the norm $\|\mid\|$. Using this result, we prove that, in most situations, if an element $T$ is in $\mathrm{K}=\overline{\operatorname{co}}^{\mathrm{w}}\left\{\delta(u) u^{*} \mid u\right.$ unitary element in $N\} \subset M$ and implements $\delta$ on $N$, then it is in $\mathscr{J}(M)$. From this we easily get the proof of the theorem for finite type I (under the local compatibility condition) and properly infinite algebras and also reduce the remaining type $\mathrm{II}_{1}$ case to the situation when $N$ is separable and $M$ is countably decomposable. Moreover, by using the Ryll-Nardzewski fixed point theorem in the same' way it is used to prove the Kadison-Sakai theorem on derivations of von Neumann algebras, we make the reduction to the case when $N^{\prime} \cap M$ contains no finite projections of $M$.

Finally, we prove the type $\mathrm{II}_{1}$ case under the above assumptions: To construct a candidate for the operator $K \in \mathscr{J}(M)$ implementing $\delta$ on $N$, we show that $N$ has an approximately finite-dimensional type II $_{1}$ von Neumann subalgebra $R \subset N$ which contains a maximal abelian *-subalgebra $A$ of $N$ such that $A^{\prime} \cap M$ contains no finite projections of $M$. The proof of this fact is inspired by [6].

We then deduce that there exists $K \in \mathscr{J}(M)$ implementing $\delta$ on $A$, and the rest of the proof shows that in fact this $K$ implements $\delta$ on all $N$. To this end we proceed by contradiction, following the lines of the proof in [7]. The assumption $\delta_{0}=\delta-\operatorname{ad} K \neq 0$ shows that $\delta_{0}(v) \neq 0$ for some unitary element $v \in N$. Then, with the help of $A$ and $v$ and using some technical devices similar to 2.1 in [7], we construct a sequence of abelian subalgebras $A_{n}$ in $N$ on which $\delta_{0}$ behaves as badly as possible. More precisely, we construct the algebras $A_{n}$ together with some finite projections $e_{n} \in M$ so that if we consider $M$ as acting on $\mathrm{L}^{2}(M, \varphi)$, then the compressions of $\delta_{0} \mid A_{n}$ to the spaces $\overline{A_{n} e_{n}} \subset \mathrm{L}^{2}(M, \varphi)$ are spatially isomorphic to a sequence of derivations $\delta_{n}: \mathrm{L}^{\infty}(\mathbb{T}, \mu) \mapsto \mathscr{B}\left(\mathrm{L}^{2}(\mathbb{T}, \mu)\right)$. We do this in such a way that the derivations $\delta_{n}$ behave more and more like ad $\mathrm{P}_{\mathrm{H}^{2}}$ and, moreover, so that by the continuity result the limit ad $\mathrm{P}_{\mathrm{H}^{2}}$ follows so-normic continuous. This is easily seen to be a contradiction. We mention that the construction of the finite projections $e_{n}$, which doesn't appear in [7], is essential here and carries most of the technical difficulties of passing from the case $M=\mathscr{B}(\mathscr{H})$ to the general case. In fact the reader will note that, although the 
proof of Theorem 1.1 is inspired in certain places by [3] and [7], our approach is rather new even when particularized to the case $M=\mathscr{B}(\mathscr{H})$.

It is our feeling that the new techniques we introduce here to deal with the case $M$ is of type $\mathrm{II}_{\infty}$ may also be used to prove Voiculescu or Andersen stability type theorems obtained when replacing $\mathscr{B}(\mathscr{H})$ by a type $\mathrm{II}_{\infty}$ factor $M$ and $\mathscr{K}(\mathscr{H})$ by $\mathscr{J}(M)$.

As the referee of the first version of this paper pointed out to us, the paper contained an error in one of the preliminary considerations, a fact that actually made that proof of Theorem 1.1 correct only in the case where the semifinite algebra $M$ had atomic center. We deeply thank the referee for pointing this out to us. However, in order to make the proof of 1.1 work in the generality presented in this paper, we only had to modify the definition of the norm I|| III and to adapt accordingly some of the statements and proofs in the preliminary section 2, a matter that only affected their form, not their spirit. In turn, the fact that in certain situations the problem has a negative answer seems to us of even more interest and clearly deserves further investigation. In particular, our Theorem 1.2 shows that one-parameter versions of classical derivation problems (or higher cohomological problems) may have negative answers.

\section{Some preliminaries.}

2.1. Let $M$ be a semifinite von Neumann algebra. Assume $M$ has countable decomposable (or countable type) center $\mathscr{Z}(M)$ and let $\psi$ be a normal faithful state on $\mathscr{Z}(M)$, fixed from now on. We will associate to $\psi$ a normal semifinite faithful trace $\varphi$ on $M$ in the following way:

Let $M$ be decomposed into a direct sum as $M=\bigoplus_{i \in I}\left(M_{i} \otimes \mathscr{B}\left(\mathscr{H}_{i}\right)\right)$, where $M_{i}$ are finite von Neumann algebras and $\operatorname{dim} \mathscr{H}_{i} \neq \operatorname{dim} \mathscr{H}_{j}$ for $i \neq j$. Let $\mathscr{Z}_{i}=\mathscr{Z}\left(M_{i}\right)$ be the center of $M_{i}$. Then $\mathscr{Z}(M)$ is naturally isomorphic to $\oplus \mathscr{Z}_{i}$. On each $M_{i}$ there is a unique normal finite faithful trace $\varphi_{i}$ which equals $\varphi$ when restricted to $\mathscr{Z}_{i}$ (here $\mathscr{Z}_{i}$ is regarded as a subalgebra of $\oplus \mathscr{Z}_{i}=\mathscr{Z}(M)$ in the obvious way). Thus there exists a unique normal semifinite faithful trace $\varphi$ on $M$ which equals $\varphi_{i} \otimes \operatorname{Tr}$ on $M_{i} \bar{\otimes} \mathscr{B}\left(\mathscr{H}_{i}\right)$, where $\operatorname{Tr}$ is the usual trace on $\mathscr{B}\left(\mathscr{H}_{i}\right)$.

We denote $M_{\varphi}=\left\{x \in M \mid \varphi\left(x^{*} x\right)<\infty\right\}$ and, for $x \in M,\|x\|_{\varphi}=\varphi\left(x^{*} x\right)^{1 / 2}$. Let $\mathscr{H}_{\varphi}$ be the Hilbert space completion of $M_{\varphi}$ in the norm \|\|$_{\varphi} . M$ will always be regarded in its standard representation, acting on $\mathscr{H}_{\varphi}$ by left multiplication. The usual uniform norm of an operator in $M$ will be denoted \|\| .

Note that if $e \in M$ is a finite projection, then we do not necessarily have $e \in M_{\varphi}$ (actually, this implication holds true only in the case where the properly infinite part of $M$ has finite-dimensional center). However, we clearly have

2.1.1. If $e \in M$ is an finite projection, then there is an increasing sequence of central projections $p_{n} \in \mathscr{Z}(M)$, so that $p_{n} \uparrow 1$ and $e p_{n} \in M_{\varphi}$ for all $n$.

2.2. We set $M_{\varphi, \psi}^{1}=\left\{x \in M_{\varphi} \mid\|x\| \leqslant 1, \varphi\left(x^{*} x p\right) \leqslant \psi(p)\right.$ for all $\left.p \in \mathscr{Z}(M)\right\}$. Although we will not use any reduction theory argument in this paper, it may be helpful for the reader to note that if $M$ is regarded as a measurable field of 
(semifinite) von Neumann factors, then, roughly speaking, a projection is in $M_{\varphi, \psi}^{1}$ if in each point it has dimension $\leqslant 1$.

The following properties of $M_{\varphi, \psi}^{1}$ will be frequently used:

2.2.1. If $T \in M,\|T\| \leqslant 1$, then $T M_{\varphi, \psi}^{1} \subset M_{\varphi, \psi}^{1}$ and $M_{\varphi, \psi}^{1} T \subset M_{\varphi, \psi}^{1}$.

2.2.2. If $e_{0}, e$ are projections in $M$ with $e_{0} \prec e$ and $e \in M_{\varphi, \psi}^{1}$, then $e_{0} \in$ $M_{\varphi, \psi}^{1}$.

2.2.3. If $x \in M_{\varphi, \psi}^{1}$, then $x^{*} \in M_{\varphi, \psi}^{1}$ and $|x| \in M_{\varphi, \psi}^{1}$.

2.2.4. If $f$ is a nonzero projection in $M$, then there exists a projection $e_{0} \neq 0$ in $M_{\varphi, \psi}^{1}$ with $e_{0} \leqslant f$. If, in addition, $e$ is properly infinite with central support $p$, then $e_{0}$ may be chosen so as to have central support $p$ and so that $\varphi\left(e_{0}\right)=\psi(p)$.

Properties 2.2.1-2.2.3 are trivial consequences of the definitions. To prove 2.2.4 it is sufficient to consider the case $M=M_{0} \bar{\otimes} \mathscr{B}(\mathscr{H})$, where $M_{0}$ is finite with center $\mathscr{Z}\left(M_{0}\right)=\mathscr{Z}(M)=\mathscr{Z}, \psi$ is a normal faithful state on $\mathscr{Z}$, and $\varphi=\tau \otimes \mathrm{Tr}$, where $\tau$ is the unique trace on $M_{0}$ which equals $\psi$ when restricted to $\mathscr{Z}\left(M_{0}\right)=\mathscr{Z}$. Let $e_{0}^{\prime}$ be a minimal projection of $\mathscr{B}(\mathscr{H})$ and $e_{0}=1 \otimes e_{0}^{\prime}$. By the comparison theorem there exists a central projection $p \in \mathscr{Z}$ such that $e_{0} p \prec f p$ and $e_{0}(1-p) \succ f(1-p)$. Thus, in particular, if $f$ is properly infinite, then $f(1-p)$ $=0$ so that $0 \neq e_{0} p \prec f p=f$ and in fact $p$ equals the central support of $f$ (because $e_{0}$ has central support one). Thus we always have a nonzero projection $e$ under $f$ in $M_{\varphi, \psi}^{1}$, and if, in addition, $f$ is properly infinite, then $e$ may be chosen to have the trace equal to the trace of $e_{0} p$, i.e., $\varphi(e)=\varphi\left(e_{0} p\right)=\psi(p)$.

2.3. Definition. For $T \in M$ we put $\|T\| \|=\sup \left\{\|T x\|_{\varphi} \mid x \in M_{\varphi, \psi}^{1}\right\}$. This is clearly a norm on $M$. It will play an important role in the sequel. Note that $\|T\| \leqslant\|T\|$ and that the equality holds if $M=\mathscr{B}(\mathscr{H})$ but fails if $M$ is nonatomic.

The next few properties are easy consequences of the definitions and of the properties of $M_{\varphi, \psi}^{1}$.

2.3.1. If $T_{1}, T_{2}, T \in M$, then $\left\|T_{1} T T_{2}\right\|\|\leqslant\| T_{1}\|\| T\|\| T_{2} \|$ and $\|T\|\|=\| T^{*}\|\|=$ ||| $|T|||$.

2.3.2. If $T \in M$ and $\left\{p_{n}\right\}_{n}$ are disjoint central projections in $M$, then $\left.\left\|T \sum_{n} p_{n}\right\|\right|^{2}=\Sigma_{n}\left\|T p_{n}\right\|^{2}$.

2.3.3. If $T, T_{0} \in M,\left\|T_{0}\right\| \leqslant 1$, and ' $\|T\|=\left\|T T_{0}\right\| \mid$, then $\|T p\|=\left\|T T_{0} p\right\| \mid$ for any central projection $p \in \mathscr{Z}(M)$.

2.3.4. If $f \in M$ is a properly infinite projection with central support $p \in$ $\mathscr{Z}(M)$, then $\|f \mid\|=\psi(p)^{1 / 2}$.

2.4. We denote by $\mathscr{J}(M)$ the norm closed two-sided ideal of $M$ generated by the finite projections of $M$. Thus an element $T \in M$ is in $\mathscr{J}(M)$ if and only if all the spectral projections $E_{[t, \infty)}(|T|)$ of $|T|$, corresponding to intervals $[t, \infty)$ with 
$t>0$, are finite projections. Alternatively, $\mathscr{J}(M)$ may be characterized as follows:

2.4.1. $K \in \mathscr{J}(M)$ if and only if, given any $\varepsilon>0$, there is a $K_{0} \in M_{\varphi}$ and a projection $p_{0} \in \mathscr{Z}(M)$ such that $\psi\left(p_{0}\right) \geqslant 1-\varepsilon, K_{0} p_{0}=K_{0}$ and $\left\|K p_{0}-K_{0}\right\|<\varepsilon$.

Indeed, assume $K \in \mathscr{J}(M)$ and let $e=E_{[\varepsilon, \infty)}(|K|)$. Then $e$ is a finite projection of $M$, so that by 2.1 .1 there exists a projection $p_{0} \in \mathscr{Z}(M)$ so that $\psi\left(p_{0}\right) \geqslant 1-\varepsilon$ and $\varphi\left(e p_{0}\right)<\infty$. Let $K_{0}=K e p_{0}$. Then $\varphi\left(K_{0}^{*} K_{0}\right) \leqslant\|K\|^{2} \varphi\left(e p_{0}\right)$ $<\infty$ and clearly $\left\|K p_{0}-K_{0}\right\|<\varepsilon$.

The other implication is trivial and, in fact, will not be needed in the sequel.

2.5. Let $K \in \mathscr{J}(M)$ and $\left\{e_{n}\right\}_{n}$ be a sequence of mutually orthogonal projections in $M$. If $M=\mathscr{B}(\mathscr{H})$, then it follows that $\left\|K e_{n}\right\| \mapsto 0$ and $\left\|e_{n} K\right\| \mapsto 0$. This is no longer true for general $M$, but still we have $\left\|K e_{n}\right\| \mapsto \mapsto 0$ and $\left\|\mid e_{n} K\right\| \| \mapsto 0$. Indeed, to prove this, since $K$ is a linear combination of four positive elements in $\mathscr{J}(M)$, we may assume $K$ is positive and $K \leqslant 1$. Let $\varepsilon>0$ and $p \in \mathscr{Z}(M)$, $K_{0} \in \mathscr{J}(M)_{+}$such that $\varphi\left(K_{0}^{2}\right)<\infty,\left\|K p-K_{0}\right\|<\varepsilon / 3$, and $\psi(p) \geqslant$ $1-\varepsilon / 3$ as in 2.4.1. Since $e_{n}$ tend weakly to zero, we have $\left\|K_{0} e_{n}\right\|_{\varphi}^{2}=\left\|e_{n} K_{0}\right\|_{\varphi}^{2}=$ $\varphi\left(e_{n} K_{0}^{2}\right) \mapsto 0$. But if $x \in M_{\varphi, \psi}^{1}$, then we have

$$
\begin{aligned}
\left\|K e_{n} x\right\|_{\varphi} & \leqslant\left\|K_{0} e_{n} x\right\|_{\varphi}+\left\|\left(K-K_{0}\right) e_{n} p x\right\|_{\varphi}+\left\|\left(K-K_{0}\right) e_{n}(1-p) x\right\|_{\varphi} \\
& \leqslant\left\|K_{0} e_{n}\right\|_{\varphi}+\left\|K p-K_{0}\right\|+\psi(1-p) \leqslant\left\|K_{0} e_{n}\right\|_{\varphi}+2 \varepsilon / 3
\end{aligned}
$$

so that if $n$ is big enough, then $\left\|K e_{n} x\right\|_{\varphi}<\varepsilon$ independently on $x \in M_{\varphi, \psi}^{1}$. Thus $\left\|K e_{n} \mid\right\| \mapsto 0$ and, similarly, $\left\|e_{n} K\right\| \| \mapsto 0$.

2.6. If $T \in M$, we denote by $\|T\|_{\text {ess }}=\inf \{\|T-K\| \| \mid K \in \mathscr{J}(M)\}$. Note that if $T \notin \mathscr{J}(M)$, then $\|T\|_{\text {ess }}>0$. Indeed, if $T \notin \mathscr{J}(M)$, then there exists $t>0$ such that $E_{[t, \infty)}(|T|)$ is an infinite projection. Thus there exists a sequence of mutually orthogonal, mutually equivalent infinite projections $\left\{f_{n}\right\}_{n}$ with $0 \neq f_{n}$ $\leqslant E_{[t, \infty)}(|T|)$. For each $n$ we take $0 \neq e_{n} \leqslant f_{n}, e_{n} \in M_{\varphi, \psi}^{1}, e_{n}$ mutually equivalent. Thus if $K \in \mathscr{J}(M)$, we get by $2.5\|T-K\|\left\|\lim _{n} \sup \right\|(T-K) e_{n} \|_{\varphi}=$ $\lim _{n} \sup \left\|T e_{n}\right\|_{\varphi} \geqslant t\left\|e_{1}\right\|_{\varphi}$, which shows that $\|T\|\left\|_{\text {ess }} \geqslant t\right\| e_{1} \|_{\varphi}>0$.

In fact, in certain simple situations this norm can be computed.

2.6.1. If $f$ is a properly infinite projection of central support $p$, then $\|f \mid\|_{\text {ess }}=\psi(p)^{1 / 2}=\|\| f \|$. More generally, if $T \in M, T \geqslant 0$ is of the form $T=\sum_{i=1}^{n} c_{i} f_{i}$ for some $c_{i}>0$ and properly infinite mutually orthogonal projections $f_{i}$ of the same central support $p$, then $\|T\|_{\text {ess }}=\left(\max \left\{c_{i}\right\}\right) \psi(p)^{1 / 2}$.

Indeed we have $c_{k} f_{k} \leqslant T \leqslant\left(\max \left\{c_{i}\right\}\right) \sum_{j} f_{j}$ for all $k$, which shows that the first part implies the second. Now the first part follows by taking a sequence of mutually orthogonal, mutually equivalent projections $e_{n} \in M_{\varphi, \psi}^{1}$ under $f$ so that 
each $e_{n}$ has central support $p$ and so that $\varphi\left(e_{n}\right)=\psi(p)$. Then for any $K \in \mathscr{J}(M)$ we get $\|f-K\|\left\|\lim _{n} \sup \right\|(f-K) e_{n} \|_{\varphi}=\psi(p)^{1 / 2}$.

Let us also note that we have for the norm \|\|$\|_{\text {ess }}$ similar properties as the properties 2.3.2 and 2.3.3 of the norm $\|\mid\|$.

2.6.2. If $T, T_{0} \in M,\left\|T_{0}\right\| \leqslant 1$, and $\|T \mid\|_{\text {ess }}=\left\|T T T_{0}\right\|_{\text {ess }}$, then $\|T p\| \|_{\text {ess }}=$ $\left\|T T_{0} p\right\|_{\text {ess }}$ for any central projection $p \in \mathscr{Z}(M)$.

2.6.3. If $\left\{p_{n}\right\}_{n}$ are disjoint central projections in $M$, then $\left\|T \sum p_{n}\right\|_{\text {ess }}^{2}=$ $\Sigma\left\|T p_{n}\right\|_{\text {ess }}^{2}$.

2.7. The norms |||| $\mid$ and || $\mid \|_{\text {ess }}$ will play a similar role in this paper as the uniform and usual essential norms do in the proof of the case $M=\mathscr{B}(\mathscr{H})$ in [3] and [7]. For our general problem these norms have all the advantages but one: for two operators of disjoint right and left supports the norm of their sum does not equal the maximum of their norms (as do the uniform and usual essential norms for $M=\mathscr{B}(\mathscr{H})$ ). We will instead use the following weaker property of the norm ||| ||| ess.

LEMMA. If $T \in M$ and $f_{1}, f_{2}$ are mutually orthogonal projections in $M$, then there are central projections $p_{1}, p_{2} \in \mathscr{Z}(M)$ such that $p_{1}+p_{2}=1$ and $\left\|\mid f_{i} T f_{i} p_{i}\right\|_{\mathrm{ess}}=\|\|\left(f_{1} T f_{1}+f_{2} T f_{2}\right) p_{i} \|_{\mathrm{ess}}, i=1,2$.

Proof. Let's first show that if $T_{1}, T_{2} \in M$, then there exists a central projection $p \in \mathscr{Z}(M)$ such that $\left\|q T_{1}\right\|_{\text {ess }} \geqslant\left\|T_{2} q\right\| \|_{\text {ess }}$ for any projection $q \in \mathscr{Z}(M)$, $q \leqslant p$, and $\left\|\mid T_{1} q\right\|_{\text {ess }} \leqslant\left\|T_{2} q\right\|_{\text {ess }}$ for any projection $q \in \mathscr{Z}(M), q \leqslant 1-p$. Indeed, by 2.6 .3 it follows that there exists a maximal projection $p$ in $\mathscr{Z}(M)$ so that for any $q \leqslant p, q \in \mathscr{P}(\mathscr{Z}(M))$, we have $\left\|T_{1} q\right\|\left\|_{\text {ess }} \geqslant\right\| T_{2} q \|_{\text {ess. }}$. Now if for some $q_{0} \leqslant 1-p$ we have $\left\|T_{1} q_{0}\right\|_{\text {ess }}>\left\|T_{2} q_{0}\right\|_{\text {ess }}$, then there exists some $p_{0} \leqslant q_{0}$ so that for any $q \leqslant p_{0}$ we have $\left\|T_{1} q\right\|_{\text {ess }}>\|\| T_{2} q \|_{\text {ess }}$; otherwise, by 2.6 .3 and a maximality argument, we get a contradiction. Thus $\left\|T_{1} q\right\|\|\leqslant\| T_{2} q\|\|$, for any $q \leqslant 1-p$.

Now we have that if $T_{1}=f_{1} T f_{1}, T_{2}=f_{2} T f_{2}$, and $p$ is the central projection corresponding to $T_{1}, T_{2}$ as above, then $p_{1}=p, p_{2}=1-p$, will satisfy the conditions. To show this, note first that since $\left|f_{1} T f_{1}+f_{2} T f_{2}\right|=\left|f_{1} T f_{1}\right|+\left|f_{2} T f_{2}\right|$, by 2.3.1 it follows that it is sufficient to prove this assertion in the case $T_{1}, T_{2} \geqslant 0$.

Let $\varepsilon>0$. Let $X_{1,2} \in M_{+}$be elements with finite spectrum so that $X_{1} \geqslant$ $T_{1}, X_{2} \leqslant T_{2},\left\|X_{1}-T_{1}\right\|<\varepsilon / 2,\left\|X_{2}-T_{2}\right\|<\varepsilon / 2$. Since $\left\|T_{1} q\right\|_{\text {ess }} \geqslant\|\| T_{2} q\|\|_{\text {ess }}$ for all $q \leqslant p$, we also have $\left\|X_{1} q\right\|_{\text {ess }} \geqslant\left\|X_{2} q\right\| \|_{\text {ess }}$ for all $q \leqslant p$. Moreover, by subtracting a compact operator from each $X_{j}$, if necessary, we may assume $X_{j}=\sum_{i} c_{i}^{j} f_{i}^{j}$, where $c_{i}^{j}>0$ and $f_{i}^{j}$ are properly infinite, mutually orthogonal projections for all $i, j$. Assume in addition that all $f_{i}^{j}$ have the same central support. Then by 2.6 .1 we have $\left\|\left(X_{1}+X_{2}\right) p\right\|\left\|_{\text {ess }}=\right\|\left\|X_{1} p\right\|_{\text {ess }}$ and since $\left\|\left|f_{1} T f_{1} p\left\|_{\text {ess }}+\varepsilon / 2 \geqslant\right\|\left\|X_{1} p\right\|_{\text {ess }}=\|\|\left(X_{1}+X_{2}\right) p\right|\right\|_{\text {ess }} \geqslant\|\|\left(f_{1} T f_{1}+f_{2} T f_{2}\right) p\|\|_{\text {ess }}$ $-\varepsilon / 2$, tending with $\varepsilon$ to zero, we get the result (the reverse inequality is trivial) in the case where all $f_{i}^{j}$ have the same central support. Now the general case reduces immediately to this one by 2.6 .3 .

Q.E.D. 
2.8. Since the norm \|\|$\|$ is a supremum of vector norms, it is inferior semicontinuous with respect to the weak operator topology. Indeed, if $T_{i}$ tends in the weak operator topology to $T$, then $\|T \xi\| \leqslant \lim _{i} \sup \left\|T_{i} \xi\right\|$, so that

$$
\begin{aligned}
\|T\| & =\sup \left\{\|T x\|_{\varphi} \mid x \in M_{\varphi, \psi}^{1}\right\} \leqslant \lim _{i} \sup \left(\sup \left\{\left\|T_{i} x\right\|_{\varphi} \mid x \in M_{\varphi, \psi}^{1}\right\}\right. \\
& =\lim _{i} \sup \left\|T_{i}\right\| .
\end{aligned}
$$

2.9. We now prove a version of Johnson and Parrott's trick in [3].

LEMMA. Let $N \subset M$ be a von Neumann algebra and $T \in M$ such that $(\operatorname{ad} T)(N) \subset \mathscr{J}(M)$ and $T \notin \mathscr{J}(M)$. Suppose the set $\mathscr{P}=\left\{f \in \mathscr{P}(N)||\|f T f\|_{\text {ess }}\right.$ $\left.=\|\mid T\|_{\text {ess }}\right\}$ contains no minimal projections. Then there exist $a c>0$ and $a$ sequence of mutually orthogonal projections $\left\{e_{n}\right\}_{n}$ in $N$ such that $\left\|e_{n} T e_{n}\right\| \|$ for all $n$.

Proof. Let $\mathscr{F}$ be a maximal chain in $\mathscr{P}$ and let $f_{0}=\inf \mathscr{F}$. Since $\mathscr{P}$ has no minimal projections, $f_{0} \notin \mathscr{P}$. Thus $c=\left(\|\mid T\|_{\text {ess }}-\|\| f_{0} T f_{0} \|_{\text {ess }}\right) / 2>0$. Then the chain $\mathscr{F}^{\prime}=\left\{f-f_{0} \mid f \in \mathscr{F}\right\}$ decreases to zero, and since

$$
\begin{aligned}
\left\|\left(f-f_{0}\right) T\left(f-f_{0}\right)\right\|_{\text {ess }}+\left\|f_{0} T f_{0}\right\|_{\text {ess }} & \geqslant\left\|\left(f-f_{0}\right) T\left(f-f_{0}\right)+f_{0} T f_{0}\right\|_{\text {ess }} \\
& =\|\| f T f\|\|_{\text {ess }}=\|T \mid\|_{\text {ess }},
\end{aligned}
$$

it follows that $\left\|f^{\prime} T f^{\prime}\right\| \|_{\text {ess }} \geqslant 2 c$ for any $f^{\prime} \in \mathscr{F}^{\prime}$.

We can now construct recursively the required sequence $\left\{e_{n}\right\}_{n \in \mathbb{N}}$. Assume $f_{1}^{\prime}, \ldots, f_{n}^{\prime}$ are $n$ projections in $\mathscr{F}^{\prime}$ with $\left\|\left(f_{k}^{\prime}-f_{k-1}^{\prime}\right) T\left(f_{k}^{\prime}-f_{k-1}^{\prime}\right)\right\| \geqslant c$, $n \geqslant k>1$. Since $\mathscr{F}^{\prime}$ is a chain decreasing to zero, by the inferior semicontinuity of the norm \|\|$\|$ it follows that there exists a projection $f_{n+1}^{\prime} \in \mathscr{F}^{\prime}$ with $f_{n+1}^{\prime} \leqslant f_{n}^{\prime}$ such that

$$
\left\|\left(f_{n}^{\prime}-f_{n+1}^{\prime}\right) T\left(f_{n}^{\prime}-f_{n+1}^{\prime}\right)\right\| \geqslant\left\|f_{n}^{\prime} T f_{n}^{\prime}\right\| / / 2 .
$$

Thus $\left\|f_{n}^{\prime} T f_{n}^{\prime}\right\|\|\geqslant\| f_{n}^{\prime} T f_{n}^{\prime} \|_{\text {ess }} \geqslant 2 c$, and consequently

$$
\left\|\left(f_{n}^{\prime}-f_{n+1}^{\prime}\right) T\left(f_{n}^{\prime}-f_{n+1}^{\prime}\right)\right\| \mid \geqslant c,
$$

so that $e_{n}=f_{n+1}^{\prime}-f_{n}^{\prime}$ will do.

Q.E.D.

2.10. Let now $M$ be an arbitrary semifinite von Neumann algebra and $N \subset M$ a weakly closed *-subalgebra of it. Let $\delta: N \mapsto \mathscr{J}(M)$ be a derivation. By [3] $\delta$ is norm continuous and by [2] it is weakly continuous. Let $p$ be the unit of $N$ and $K=\delta(p) p-p \delta(p) \in \mathscr{J}(M)$. Then $K p-p K=\delta(p) p-2 p \delta(p) p+$ $p \delta(p)=(\delta(p)-p \delta(p))-\left(2 \delta\left(p^{2}\right) p-2 \delta(p) p^{2}\right)+p \delta(p)=\delta(p)$ so that $(\delta-\operatorname{ad} K)(p)=0$ and $(\delta-\operatorname{ad} K)(x)=(\delta-\operatorname{ad} K)(p x p)=p(\delta-\operatorname{ad} K)$ $(x) p$, which shows that $\delta-$ ad $K$ takes values in $p M p$.

This shows that in order to prove Theorem 1.1, we may assume the weakly closed *-subalgebra $N \subset M$ has the same unit as $M$, i.e., $N$ is a von Neumann 
subalgebra of $M$. Therefore, in all the rest of the paper the subalgebra $N$ will be considered to have the same unit as $M$.

2.11. Let $\left\{p_{i}\right\}_{i \in I}$ be a family of mutually orthogonal projections in the center of $M$ with $\sum_{i} p_{i}=1$. Assume that for each $i$ there exists $K_{i} \in \mathscr{J}(M) p_{i}=$ $\mathscr{J}\left(M_{p_{i}}\right)$ such that $\delta(x) p_{i}=\operatorname{ad} K_{i}(x)$ and $\left\|K_{i}\right\| \leqslant 2\|\delta\|$ for all $x \in N$. Then $K=\sum_{i \in I} K_{i}$ is in $\mathscr{J}(M)$ and $\delta=\operatorname{ad} K$ on $N$.

Since in a semifinite von Neumann algebra there exist mutually orthogonal central projections $p_{i}$ with $\sum p_{i}=1$ such that each $\mathscr{Z}(M) p_{i}$ is countable decomposable (or, equivalently, has a normal faithful state), it follows by the above observation that to prove Theorem 1.1 for general $M$ it is sufficient to prove it for each $M_{p_{i}}$, i.e., under the assumption that $\mathscr{Z}(M)$ is of countable type. Thus we may and will assume in the rest of the paper that $M$ has countable decomposable center $\mathscr{Z}(M)$, that $\psi$ is a normal faithful state on $\mathscr{Z}(M)$, and that $\varphi$ is the unique normal faithful trace on $M$ associated to $\psi$ as in 2.1. The reader will note that each time we get a $K \in \mathscr{J}(M), \delta=$ ad $K$ for $M$ of countable type, we also have $\|K\| \leqslant 2\|\delta\|$.

2.12. Let $N_{0} \subset N$ be a finite-dimensional von Neumann subalgebra of $N, \mathscr{U}_{0}$ the unitary compact group of $N_{0}$, and $\lambda$ the normalized Haar measure on $\mathscr{U}_{0}$.

Then $K=\int \delta(u) u^{*} \mathrm{~d} \lambda(u) \in \mathscr{J}(M)$ satisfies for any $u_{0} \in \mathscr{U}_{0}$ :

$$
\begin{aligned}
K u_{0}-u_{0} K & =\int \delta(u) u^{*} u_{0} \mathrm{~d} \lambda(u)-\int u_{0} \delta(u) u^{*} \mathrm{~d} \lambda(u) \\
& =\int \delta(u)\left(u_{0}^{*} u\right)^{*} \mathrm{~d} \lambda(u)-\int u_{0} \delta(u) u^{*} \mathrm{~d} \lambda(u) \\
& =\int \delta\left(u_{0} u\right) u^{*} \mathrm{~d} \lambda(u)-\int u_{0} \delta(u) u^{*} \mathrm{~d} \lambda(u) \\
& =\delta\left(u_{0}\right) \int \mathrm{d} \lambda(u)+\int u_{0} \delta(u) u^{*} \mathrm{~d} \lambda(u)-\int u_{0} \delta(u) u^{*} \mathrm{~d} \lambda(u) \\
& =\delta\left(u_{0}\right)
\end{aligned}
$$

Thus $(\delta-\operatorname{ad} K)\left(x_{0}\right)=0$ for any $x_{0} \in N_{0}$. In particular, this shows that if $N$ is a finite direct sum, then to prove 1.1 for $N \subset M$ it is sufficient to prove it for each summand.

3. The atomic abelian case. In this section we prove Theorem 1.1 in the case $N$ is isomorphic to the algebra $\ell^{\infty}(I)$ for a set $I$ of arbitrary cardinality.

To do this, let $\left\{e_{i}\right\}_{i \in I}$ be the minimal projections of $N=\ell^{\infty}(I)$ and note first that the series $\sum_{i \in I} \delta\left(e_{i}\right) e_{i}$ is convergent in the strong operator topology. Indeed, 
the sequence is bounded because if $e_{1}, e_{2}, \ldots, e_{n} \in\left\{e_{i}\right\}_{i \in I}$, then

$$
\begin{aligned}
\sum_{k=1}^{n} \delta\left(e_{k}\right) e_{k} & =\sum_{k, l=1}^{n} \int z_{k} \bar{z}_{l} \delta\left(e_{k}\right) e_{l} \mathrm{~d} \lambda(z) \\
& =\int \delta\left(\sum_{k=1}^{n} z_{k} e_{k}\right)\left(\sum_{l=1}^{n} \bar{z}_{l} e_{l}\right) \mathrm{d} \lambda(z),
\end{aligned}
$$

where $\lambda$ is the normalized Haar measure on the torus $\mathbb{T}^{n}$ and $z=\left(z_{1}, z_{2}, \ldots, z_{n}\right)$ $\in \mathbb{T}^{n}$, so that

$$
\left\|\sum_{k=1}^{n} \delta\left(e_{k}\right) e_{k}\right\| \leqslant \int\left\|\delta\left(\sum_{k=1}^{n} z_{k} e_{k}\right)\left(\sum_{l=1}^{n} \bar{z}_{l} e_{l}\right)\right\| \mathrm{d} \lambda(z) \leqslant\|\delta\| .
$$

Now if $M$ is normally represented on some Hilbert space $\mathscr{H}, \xi \in \mathscr{H}$, and $\varepsilon>0$, then there exists a finite set $I_{0} \subset I$ such that $\left\|\xi-\left(\sum_{i \in I_{0}} e_{i}\right) \xi\right\|<\varepsilon$, and thus for any finite set $J_{0} \subset I$ with $J_{0} \cap I_{0}=\varnothing$ we have

$$
\left\|\sum_{i \in J_{0}} \delta\left(e_{j}\right) e_{j} \xi\right\| \leqslant \varepsilon\|\delta\|+\left\|\left(\sum_{j \in J_{0}} \delta\left(e_{j}\right) e_{j}\right)\left(\sum_{i \in I_{0}} e_{i}\right) \xi\right\|=\varepsilon\|\delta\|,
$$

which shows that $\sum_{i \in I} \delta\left(e_{i}\right) e_{i} \xi$ is convergent for any $\xi \in \mathscr{H}$.

Let $T=\sum_{i \in I} \delta\left(e_{i}\right) e_{i}$. Since $\delta$ is a derivation and $\left(\sum_{i \in I} \delta\left(e_{i}\right) e_{i}\right) e_{i_{0}}=\delta\left(e_{i_{0}}\right) e_{i_{0}}$, we have

$$
\begin{aligned}
T e_{i_{0}}-e_{i_{0}} T & =\delta\left(e_{i_{0}}\right) e_{i_{0}}-\sum_{i \in I} e_{i_{0}} \delta\left(e_{i}\right) e_{i} \\
& =\delta\left(e_{i_{0}}\right) e_{i_{0}}-\sum_{i \in I} \delta\left(e_{i_{0}} e_{i}\right) e_{i}+\delta\left(e_{i_{0}}\right) \sum_{i \in I} e_{i} \\
& =\delta\left(e_{i_{0}}\right) e_{i_{0}}-\delta\left(e_{i_{0}}\right) e_{i_{0}}+\delta\left(e_{i_{0}}\right)=\delta\left(e_{i_{0}}\right) .
\end{aligned}
$$

Since both $\delta$ and ad $T$ are weakly continuous on $N$ and the linear span of $\left\{e_{i}\right\}_{i \in I}$ is weakly dense in $N=\ell^{\infty}(I)$, it follows that $\delta=\operatorname{ad} T$ on $N$.

We show that $T$ is in $\mathscr{J}(M)$. Suppose $T \notin \mathscr{J}(M)$. Let

$$
\mathscr{P}=\left\{f \in \mathscr{P}(N)\left|\|f T f\|_{\text {ess }}=\|\mid\| T \|_{\text {ess }}\right\} .\right.
$$

Then $\mathscr{P}$ contains no minimal projections, because if $e \in \mathscr{P}$ is a minimal projection of $\mathscr{P}$ and $e_{0} \leqslant e$ is a minimal projection of $N$, then $e_{0} T e_{0}=0$ (by the definition of $T$ ), so that $e-e_{0} \in \mathscr{P}$, a contradiction. Thus by 2.9 there exist 
$c>0$ and a sequence of mutually orthogonal projections $\left\{f_{n}\right\}_{n \in \mathbb{N}}$ in $N$ such that

$$
\left\|f_{n} T f_{n}\right\| \geqslant c \text { for all } n \text {. }
$$

Moreover, by the inferior semicontinuity of the norm || ||| we may assume each projection $f_{n}$ is the sum of a finite set $J_{n} \subset J$ of minimal projections in $N$. But by (*) we have

$$
T f_{n}=\sum_{j \in J_{n}} \delta\left(e_{j}\right) e_{j}=\int \delta\left(\sum_{i \in J_{n}} z_{i} e_{i}\right)\left(\sum_{j \in J_{n}} \bar{z}_{j} e_{j}\right) \mathrm{d} \lambda(z),
$$

so that

$$
\int\left\|f_{n} \delta\left(\sum_{i \in J_{n}} z_{i} e_{i}\right)\left(\sum_{j \in J_{n}} \bar{z}_{j} e_{j}\right) f_{n}\right\| \mathrm{d} \lambda(z) \geqslant\left\|f_{n} T f_{n}\right\| \| c
$$

which implies that for some $u_{n}=\sum_{i \in J_{n}} z_{i} e_{i}$,

$$
\left\|f_{n} \delta\left(u_{n}\right) u_{n}^{*} f_{n}\right\| \geqslant c .
$$

Now let $u=\sum_{n \in \mathbb{N}} u_{n}$. Then, for each $n$,

$$
f_{n} \delta(u) u^{*} f_{n}=f_{n} \delta\left(f_{n} u\right) u_{n}^{*} f_{n}-f_{n} \delta\left(f_{n}\right) f_{n}=f_{n} \delta\left(u_{n}^{*}\right) u_{n} f_{n}
$$

so that

$$
\left\|f_{n} \delta(u) u^{*} f_{n}\right\|=\left|\left\|f_{n} \delta\left(u_{n}^{*}\right) u_{n} f_{n}\right\|\right| \geqslant c .
$$

Since $\delta(u) u^{*}$ is in $\mathscr{J}(M)$, by 2.5 this is a contradiction. Thus $\sum_{i \in I} \delta\left(e_{i}\right) e_{i}$ is in $\mathscr{J}(M)$, and the case $N=\ell^{\infty}(I)$ is solved.

4. The continuity result. For the next result we assume $N \subset M$ is a finite von Neumann algebra with a normal faithful finite trace $\tau, \tau(1)=1$. We denote by $\|x\|_{2}=\tau\left(x^{*} x\right)^{1 / 2}, x \in N$.

4.1. Proposition. Let $\delta: N \mapsto \mathscr{J}(M)$ be a derivation. Then $\delta$ is continuous from the unit ball of $N$ with the strong operator topology into $\mathscr{J}(M)$ with the norm |II |||.

Proof. We first prove that if $\left\{f_{n}\right\}_{n \in \mathbb{N}}$ is a sequence of projections in $N$ with $\tau\left(f_{n}\right) \mapsto 0$, then $\left\|\delta\left(f_{n}\right)\right\| \| \mapsto 0$. Suppose $\left\|\delta\left(f_{n}\right)\right\| \|$ does not converge to 0 . By taking a subsequence, if necessary, we may assume that $\left\|\delta\left(f_{n}\right)\right\| \geqslant c$ for some $c>0$ and all $n$ and that $\sum \tau\left(f_{n}\right)<\infty$. Let $g_{n}$ be the supremum of $\left\{f_{k}\right\}_{k \geqslant n}$. Then $\tau\left(g_{n}\right) \leqslant \sum_{k \geqslant n} \tau\left(f_{k}\right)$ tends to zero with $n$. Denote by $s_{n, m}$ the support of $f_{m} g_{n} f_{m}$. Then $s_{n m} \leqslant f_{m}$ and $s_{n, m}$ is majorized by $g_{n}$ and thus, $\tau$ being a trace, 
$\tau\left(s_{n m}\right) \leqslant \tau\left(g_{n}\right) \mapsto_{n} 0$ for each $m$. Since $\left\{g_{n}\right\}_{n \in \mathbb{N}}$ is decreasing, $\left\{f_{m} g_{n} f_{m}\right\}_{n \in \mathbb{N}}$ is decreasing so that $\left\{s_{n m}\right\}_{n \in \mathbb{N}}$ is decreasing for each $m$. Thus $\left\{f_{m}-s_{n m}\right\}_{n \in \mathbb{N}}$ increases to $f_{m}$, so that $\left\{\delta\left(f_{m}-s_{n m}\right)\right\}_{n \in \mathbb{N}}$ is weakly convergent to $\delta\left(f_{m}\right)$. By the inferior semicontinuity of the norm \|\|$\|$ (cf. 2.8) it follows that for a fixed $m$, if $n$ is big enough, $\left\|\left|\delta\left(f_{m}-s_{n m}\right) \|\right| \geqslant c / 2\right.$.

We may thus get by induction an increasing sequence of integers $n_{1}, n_{2}, \ldots$ such that the projections $h_{k}=f_{n_{k}}-s_{n_{k+1}, n_{k}}$ satisfy $\left\|\delta\left(h_{k}\right)\right\| \| c / 2$. These projections also satisfy $\tau\left(h_{k}\right) \leqslant \tau\left(f_{n_{k}}\right) \mapsto_{k} 0$.

Moreover, since $h_{k} \leqslant f_{n_{k}}$ and $s_{n_{k+1}, n_{k}}$ is the support of $f_{n_{k}} g_{n_{k+1}} f_{n_{k}}$, by the definition of $h_{k}$ we get

$$
h_{k} g_{n_{k+1}} h_{k}=h_{k} f_{n_{k}} g_{n_{k+1}} f_{n_{k}} h_{k} \leqslant h_{k} s_{n_{k+1}} f_{n_{k}} h_{k} \leqslant h_{k} s_{n_{k+1}, n_{k}} h_{k}=0 .
$$

Thus $h_{k} g_{n_{k+1}}=0$, in particular $h_{k} f_{n_{l}}$ for $l \geqslant k+1$, and so $h_{k} h_{l}=0$, which means that $h_{k}$ are all mutually orthogonal projections. Since we also have $\left\|\left|\delta\left(h_{k}\right)\right|\right\| \geqslant c / 2$, we obtain a contradiction, by the atomic abelian case (\$3) and 2.5.

Now we turn to the general case. Since \|\|$_{2}$ induces the strong operator topology on the unit ball of $M$, we have to show that if $\left(x_{n}\right)_{n}$ is a bounded sequence in $M$ with $\left\|x_{n}\right\| \mapsto_{2} 0$, then $\left\|\delta\left(x_{n}\right)\right\| \| \rightarrow$. It is clear that we only need to prove this implication in the case where $x_{n}$ are self-adjoint elements and $\left\|x_{n}\right\| \leqslant 1$. Moreover, since $\left\|\left|x_{n}\right|\right\|_{2}=\left\|x_{n}\right\|_{2}$, it follows that if $\left\|x_{n}\right\|_{2} \mapsto 0$, then $\left\|\left(x_{n}\right)_{+}\right\|_{2} \mapsto 0$ and $\left\|\left(x_{n}\right)_{-}\right\|_{2} \mapsto 0$, so that it is sufficient to prove that if $x_{n}$ are positive elements and $\left\|x_{n}\right\|_{2} \mapsto 0$ (equivalently, $\tau\left(x_{n}\right) \mapsto 0$ ), then $\left\|\delta\left(x_{n}\right)\right\| \| \mapsto 0$.

Let $x_{n}=\sum_{m \geqslant 1} 2^{-m} e_{m}^{n}$ be the diadic decomposition of $x_{n}$. It follows that $\tau\left(e_{m}^{n}\right) \mapsto_{n} 0$ for each $m \geqslant 1$. Let $\varepsilon>0$ and $m_{0} \geqslant 1$ so that $2^{-m_{0}} \leqslant \varepsilon / 2$. Then by the first part of the proof there exists $n_{0}$ such that for $n \geqslant n_{0},\left\|\left|\delta\left(e_{m}^{n}\right)\right|\right\|<\varepsilon / 2$ for any $m \leqslant m_{0}$. Thus, for $n \geqslant n_{0}$ we get

$$
\left\|\delta\left(x_{n}\right)\right\| \leqslant \sum_{m=1}^{m_{0}} 2^{-m}\left\|\delta\left(e_{m}^{n}\right)\right\|+\|\delta\| \sum_{m>m_{0}} 2^{-m} \leqslant \varepsilon . \quad \text { Q.E.D. }
$$

The above continuity result will enable us to reduce the theorem to more tractable situations and to prove it in several cases. We will actually use the following consequence of 4.1 .

4.2. Corollary. Let $\mathrm{K}_{\delta}=\overline{\operatorname{co}}^{\mathrm{w}}\left\{\delta(u) u^{*} \mid u\right.$ unitary element in $\left.N\right\}$. Assume $N$ is finite and countable decomposable and denote by $\tau$ a normal finite faithful trace on it, $\tau(1)=1$. Given $\beta>0$, there exists $\alpha>0$ such that if $x \in N,\|x\| \leqslant 1$, and $\|x\|_{2} \leqslant \alpha$, then

$$
\||T x \|| \leqslant \beta \quad \text { and } \quad\| x T \| \leqslant \beta \quad \text { for all } T \in \mathrm{K}_{\delta} .
$$


Proof. By the preceding proposition there exists $\alpha>0$ such that $\|y\| \leqslant 1$, $\|y\|_{2}<\alpha$, implies $\|\delta(y)\|<\beta / 3$. Since $\delta(u) u^{*} y=\delta(y)-u \delta\left(u^{*} y\right)$ and $\left\|u^{*} y\right\|_{2}$ $=\|y\|_{2}$, it follows that

$$
\left\|\delta(u) u^{*} y\right\| \leqslant\|\delta(y)\|\left|+\left\|\delta\left(u^{*} y\right)\right\|\right|<2 \beta / 3
$$

for any unitary element $u$ in $M$. By taking convex combinations of $\delta(u) u^{*}$ and using the fact that the norm $\|\mid\|$ is weak inferior semicontinuous, we get $\|T y\|<\beta$ for all $T \in \mathrm{K}_{\delta}$. Similarly, $\|y T\| \leqslant \beta$.

Q.E.D.

Actually, we will mostly use 4.1 and 4.2 through the next technical results, which show that in many cases, whenever there exists $T \in K_{\delta}$ (defined as in 4.2) with ad $T=\delta$, then $T \in \mathscr{J}(M)$.

First we consider the case when $N$ is abelian and locally compatible with $\mathscr{Z}(M)$ (in the sense of 1.1).

4.3. Proposition. Assume that the von Neumann subalgebra $N$ of $M$ is abelian and that there exist projections $\left\{e_{i}\right\}_{i \in I}$ in $N$ so that $\sum e_{i}=1$ and so that for each $i$ we have either $N e_{i} \supset \mathscr{Z}(M) e_{i}$ or $N e_{i} \subset \mathscr{Z}(M) e_{i}$. Moreover, assume that there exist projections $\left\{p_{j}\right\}_{j \in J}$ in $N$ so that $\sum p_{j}=1, N_{p_{j}}$ is of countable type for each $j$ and $\delta$ vanishes on the set $\left\{p_{j}\right\}_{j}$.

If $T \in K_{\delta}=\overline{\operatorname{co}}^{\mathrm{w}}\left\{\delta(u) u^{*} \mid u \in \mathscr{U}(N)\right\}$ is so that ad $T=\delta$, then $T \in J(M)$.

Proof. Assume $T \notin \mathscr{J}(M)$, so that $\|T\|_{\text {ess }}>0$. Let

$$
\mathscr{P}=\left\{e \in \mathscr{P}(N)\left|\||| e T e\|_{\text {ess }}=\|\| T \|_{\text {ess }}\right\} .\right.
$$

If $\mathscr{P}$ contains no minimal projections, then by 2.9 there exists a sequence of mutually orthogonal projections $\left\{e_{n}\right\}_{n}$ in $N$ so that $\left\|e_{n} T e_{n}\right\| \| c$ for some $c>0$ and all $n$.

By the inferior semicontinuity of the norm \|\|$\|$, for each $n$ we can find a projection $p_{n}$ in the von Neumann algebra generated by $\left\{p_{i}\right\}_{i \in I}$ such that $N_{p_{n}}$ is countable decomposable and

$$
\left\|e_{n} T e_{n} p_{n}\right\| \geqslant\left\|e_{n} T e_{n}\right\| / 2=c / 2 .
$$

Let $p$ be the supremum of $\left\{p_{n}\right\}_{n}$. Then $p$ belongs to $\left\{p_{i}\right\}_{i \in I}^{\prime \prime}$ (and thus $\delta(p)=0), N_{p}$ is countable decomposable and clearly

$$
\left\|\mid e_{n} T e_{n} p\right\| \| c / 2 \text { for all } n \text {. }
$$

By construction $e_{n} p$ tends strongly to zero in $N_{p}$.

If we consider $\delta^{\prime}: N_{p} \mapsto \mathscr{J}\left(M_{p}\right)$ defined by $\delta^{\prime}(x p)=\delta(x) p$, then obviously $T p \in K_{\delta^{\prime}}$. Thus by 4.2 we have $\left\|e_{n} T e_{n} p\right\| \rightarrow 0$, a contradiction.

Assume now that $\mathscr{P}$ has minimal projections and denote one of them by $e$. Assume first that $e e_{i} \neq 0$ for some $i$ with $N e_{i} \subset \mathscr{Z}(M) e_{i}$. Denote $f_{0}=e e_{i}$. Then 
we also have $N f_{0} \subset \mathscr{Z}(M) f_{0}$. It follows that for any unitary element $u \in \mathscr{U}(N)$ there is a $z_{0} \in \mathscr{U}(\mathscr{Z}(M))$ such that $u f_{0}=z_{0} f_{0}$, and we have

$$
\begin{aligned}
f_{0} \delta(u) u^{*} f_{0} & =\delta\left(f_{0}\right) f_{0}-\delta\left(f_{0} u\right) u^{*} f_{0} \\
& =\delta\left(f_{0}\right) f_{0}-\left(T f_{0} u-f_{0} u T\right) u^{*} f_{0} \\
& =\delta\left(f_{0}\right) f_{0}-\left(T z_{0} f_{0}-z_{0} f_{0} T\right) z_{0}^{*} f_{0} \\
& =\delta\left(f_{0}\right) f_{0}-\left(T f_{0}-f_{0} T\right) z_{0} z_{0}^{*} f_{0}=0 .
\end{aligned}
$$

Thus, since $T \in \overline{\operatorname{co}}^{\mathrm{w}}\left\{\delta(u) u^{*} \mid u \in \mathscr{U}(N)\right\}$, we get $f_{0} T f_{0}=0$, which implies that $e T e-\left(e-f_{0}\right) T\left(e-f_{0}\right) \in \mathscr{J}(M)$. Thus $e-f_{0} \in \mathscr{P}$, contradicting the minimality of $e$ in $\mathscr{P}$.

Now the only case left is when there is a nonempty set $I_{0} \subset I$ so that $e_{i} \leqslant e$ and $N e_{i} \supset \mathscr{Z}(M) e_{i}$ for all $i \in I_{0}$ and $\sum_{i \in I_{0}} e_{i}=e$. Fix an $i$ in $I_{0}$ with $e_{i} \neq 0$. If $N e_{i}=\mathscr{Z}(M) e_{i}$, then the first case applies and leads to a contradiction, so we may assume $N e_{i} \neq \mathscr{Z}(M) e_{i}$, and in fact we may assume there exists no $e_{i}^{\prime} \in N e_{i}$ so that $N e_{i}^{\prime}=\mathscr{Z}(M) e_{i}^{\prime}$. It then follows that there exists a projection $f_{i} \in N e_{i}$ so that $q f_{i} \notin \mathscr{Z}(M) e_{i}$ for any $q \in \mathscr{Z}(M), q \neq 0$.

Then by 2.7 it follows that there exists a projection $q_{i}^{\prime} \in \mathscr{Z}(M)$ such that

$$
\begin{gathered}
\left\|T f_{i} q_{i}^{\prime} \mid\right\|_{\mathrm{ess}}=\left\|T e_{i} q_{i}^{\prime}\right\| \|_{\mathrm{ess}}, \\
\left\|T\left(e_{i}-f_{i}\right)\left(1-q_{i}^{\prime}\right)\right\|_{\mathrm{ess}}=\left\|T e_{i}\left(1-q_{i}^{\prime}\right)\right\|_{\mathrm{ess}} .
\end{gathered}
$$

Thus if we denote by $e_{i}^{\prime}=f_{i} q_{i}^{\prime}+\left(e_{i}-f_{i}\right)\left(1-q_{i}^{\prime}\right)$, then we have $e_{i}^{\prime} \leqslant e_{i}$, $e_{i}^{\prime} \neq e_{i}, e_{i}^{\prime} \in \mathscr{P}(N)$, and by 2.3 .2 we have $\left\|T e_{i}^{\prime}\right\|_{\text {ess }}^{2}=\left\|T f_{i} q_{i}^{\prime}\right\|_{\text {ess }}^{2}+\| T\left(e_{i}-f_{i}\right)$ $\left(1-q_{i}^{\prime}\right)\left\|_{\text {ess }}^{2}=\right\| T e_{i} q_{i}^{\prime}\left\|_{\text {ess }}^{2}+\right\| T e_{i}\left(1-q_{i}^{\prime}\right)\left\|_{\text {ess }}^{2}=\right\| T e_{i} \|_{\text {ess }}^{2}$.

Denote by $e^{\prime}=\left(e-e_{i}\right)+e_{i}^{\prime}$. Then $e^{\prime} \in N, e^{\prime} \leqslant e$ and $e^{\prime} \neq e$.

Then let $q_{i} \in \mathscr{Z}(M)$ be a projection satisfying

$$
\begin{gathered}
\left\|T e_{i} q_{i}\right\|_{\mathrm{ess}}=\left\|T e q_{i}\right\| \|_{\mathrm{ess}}, \\
\left\|T\left(e-e_{i}\right)\left(1-q_{i}\right)\right\|_{\mathrm{ess}}=\left\|T e\left(1-q_{i}\right)\right\|_{\mathrm{ess}} .
\end{gathered}
$$

Then we have

$$
\begin{aligned}
\|T e\|_{\mathrm{ess}}^{2} & =\left\|T\left(e-e_{i}\right)\left(1-q_{i}\right)\right\|_{\mathrm{ess}}^{2}+\left\|T e_{i} q_{i}\right\| \|_{\mathrm{ess}}^{2} \\
& =\left\|T\left(e-e_{i}\right)\left(1-q_{i}\right)\right\|_{\mathrm{ess}}^{2}+\left\|T e_{i}^{\prime} q_{i}\right\|_{\mathrm{ess}}^{2} \\
& =\left\|T\left(e_{i}^{\prime} q_{i}+\left(e-e_{i}\right)\left(1-q_{i}\right)\right)\right\|_{\mathrm{ess}}^{2} \\
& \leqslant\left\|T\left(e_{i}^{\prime}+e-e_{i}\right)\right\|_{\mathrm{ess}}^{2}=\left\|T e^{\prime}\right\|_{\mathrm{ess}}^{2} \leqslant\|T e\|_{\mathrm{ess}}^{2} .
\end{aligned}
$$


Thus $\|T e\|_{\text {ess }}=\left\|T e^{\prime}\right\| \|_{\text {ess }}$, which again contradicts the minimality of $e$. This ends the proof of the proposition.

Q.E.D.

4.4. Proposition. Let $N \subset M$ be a von Neumann subalgebra of type $\mathrm{II}_{1}$. Assume the derivation $\delta: N \mapsto \mathscr{J}(M)$ vanishes on a set of projections $\left\{p_{i}\right\}_{i} \subset$ $\mathscr{Z}(M)$ with the property that $\sum p_{i}=1$ and $N_{p_{i}}$ is countable decomposable for all $i$. If $T \in K_{\delta}$ is such that ad $T=\delta$ on $N$, then $T \in \mathscr{J}(M)$.

Proof. Since $N$ is of type $\mathrm{II}_{1}$, there exists a decreasing sequence of projections $\left\{e_{n}\right\}_{n \geqslant 0}$ in $N$ with $e_{0}=1, e_{n+1} \sim e_{n}-e_{n+1}$ for all $n \geqslant 0$. Suppose we have shown that for some $n \geqslant 0$ we have $\left\|e_{k} T e_{k}\right\|\left\|_{\text {ess }}=\right\| T \mid \|_{\text {ess }}$ for all $k \leqslant n$. Let $u_{n}$ be a unitary element in $N$ such that $u_{n} e_{n+1} u_{n}^{*}=e_{n}-e_{n+1}$. Since $u_{n} T u_{n}^{*}-$ $T \in \mathscr{J}(M)$, we have $\left\|e_{n+1} T e_{n+1} q\right\|_{\text {ess }}=\left\|u_{n} e_{n+1} T e_{n+1} u_{n}^{*} q\right\|_{\text {ess }}=$ $\left\|u_{n} e_{n+1} u_{n}^{*} T u_{n} e_{n+1} u_{n}^{*} q\right\|_{\text {ess }}=\|\|\left(e_{n}-e_{n+1}\right) T\left(e_{n}-e_{n+1}\right) q\|\|_{\text {ess }}$ for any central projection $q \in \mathscr{Z}(M)$. Since

$$
\left\|\mid e_{n} T e_{n}\right\|\left\|_{\text {ess }}=\right\| e_{n+1} T e_{n+1}+\left(e_{n}-e_{n+1}\right) T\left(e_{n}-e_{n+1}\right) \|_{\text {ess }},
$$

by 2.7 and 2.3 .2 it follows that $\left\|\left|e_{n+1} T e_{n+1}\right|\right\|_{\text {ess }}=\|\| e_{n} T e_{n}|\||_{\text {ess. }}$. Thus $\left\|\mid e_{n} T e_{n}\right\| \|_{\text {ess }}=$ $\|T \mid\|_{\text {ess }}$ for all $n$.

Assume $T \notin \mathscr{J}(M)$, so that $\left\|e_{n} T e_{n}\right\| \|_{\text {ess }} \geqslant c>0$ for all $n$.

Then the proof continues exactly the same way as the first part of the proof of 4.3 and leads to a contradiction.

Q.E.D.

4.5. Lemma. Let $A \subset N$ be an abelian von Neumann subalgebra of $N$ and suppose there exists a decreasing sequence of nonzero projections $\left\{e_{n}\right\}_{n}$ in $A$ such that $e_{n} \downarrow 0, e_{0}=1$, and $e_{n+1}$ is equivalent to $e_{n}-e_{n+1}$ in $N$ for all $n \geqslant 0$. If $T \in \mathrm{K}_{\delta, A}=\overline{c o}^{w}\left\{\delta(u) u^{*} \mid u\right.$ unitary element in $\left.A\right\}$ is so that ad $T=\delta$ on $N$, then $T \in \mathscr{J}(M)$.

Proof. If $v_{n} \in N$ are so that $v_{n}^{*} v_{n}=e_{n+1}, v_{n} v_{n}^{*}=e_{n}-e_{n+1}$, then we have $v_{n} T-T v_{n} \in \mathscr{J}(M)$ and for any $q \in \mathscr{Z}(M)$,

$$
\begin{aligned}
\left\|e_{n+1} T e_{n+1} q\right\| \|_{\text {ess }} & =\left\|v_{n} e_{n+1} T e_{n+1} v_{n}^{*} q\right\|\left\|_{\text {ess }}=\right\|\left\|v_{n} e_{n+1} v_{n}^{*} T v_{n} e_{n+1} v_{n}^{*} q\right\|_{\text {ess }} \\
& =\left\|\left(e_{n}-e_{n+1}\right) T\left(e_{n}-e_{n+1}\right) q\right\|_{\text {ess }} .
\end{aligned}
$$

The rest of the proof is exactly as the proof of 4.4 .

Q.E.D.

We end this section by proving a useful converse to the preceding results. Note that the proof doesn't use the continuity result 4.1 .

4.6. LEMMA. Let $N$ be an arbitrary von Neumann subalgebra of $M$ and $\delta: N \mapsto \mathscr{J}(M)$ a derivation. If there exists $K \in \mathscr{J}(M)$ such that $\delta=\operatorname{ad} K$, then there exists $T \in K_{\delta}$ such that $\delta=\operatorname{ad} T$.

Proof. Assume first that $\varphi\left(K^{*} K\right)<\infty$. Let

$$
C=\overline{\mathrm{co}}^{\mathrm{w}}\left\{u K u^{*} \mid u \text { unitary element in } N\right\} \text {. }
$$


Then $\|y\|_{\varphi} \leqslant\|K\|_{\varphi}$ for all $y$ in $C$ and $C$ is a weakly compact convex subset of $M$. By the inferior semicontinuity of the norm \|\|$_{\varphi}$ it follows that there exists a unique element $y_{0} \in C$ with $\left\|y_{0}\right\|_{\varphi} \leqslant\|y\|_{\varphi}$ for all $y \in C$. Since $u y_{0} u^{*} \in C$ and $\left\|u y_{0} u^{*}\right\|_{\varphi}=\left\|y_{0}\right\|_{\varphi}$, it follows that $u y_{0} u^{*}=y_{0}$ for all unitary elements $u \in N$. Thus $y_{0} \in C \cap N^{\prime}$.

Let's now show that for arbitrary $K$ there also exists some $y \in C \cap N^{\prime}$. To this end note first that for each $n$ there exist (by 2.4) $p_{n} \in \mathscr{P}(\mathscr{Z}(M))$ and $K_{n} \in M_{\varphi} \subset \mathscr{J}(M)$ such that $\left\|K_{n}\right\| \leqslant\|K\|, K_{n} p_{n}=K_{n},\left\|K p_{n}-K_{n}\right\| \leqslant 1 / n$, and $\psi\left(p_{n}\right) \geqslant 1-1 / n$. Let $C_{n}=\overline{c o}^{\mathrm{w}}\left\{u K_{n} u^{*} \mid u\right.$ unitary element in $\left.N\right\}$ and $y_{n} \in C_{n} \cap$ $N^{\prime}$ (cf. the first part of the proof). Then the Hausdorf distance between $C_{n}$ and $p_{n} C$ satisfies

$$
\mathrm{d}\left(p_{n} C, C_{n}\right) \leqslant\left\|K p_{n}-K_{n}\right\| \leqslant 1 / n \text {. }
$$

Thus there exists $x_{n} \in C p_{n}$ so that $\left\|x_{n}-y_{n}\right\| \mapsto 0$.

Now let $y$ be a weak limit point of $\left\{y_{n}\right\}_{n}$. It follows that $y \in N^{\prime}$ (because $y_{n} \in N^{\prime}$ for each $n$ ) and $y \in C$ (because $y$ is also a limit point of $\left\{x_{n}\right\}_{n}$ ).

Now set $T=K-y$. Then $K-y \in K-C=\overline{c o}^{\text {w }}\left\{K-u K u^{*} \mid u\right.$ unitary element in $N\}=\mathrm{K}_{\delta}$ and, moreover, since $y \in N^{\prime}$, ad $T=\operatorname{ad} K=\delta$. $\quad$ Q.E.D.

5. The type I and properly infinite cases. We first prove Theorem 1.1 when $N$ is a finite type I von Neumann algebra locally compatible with $\mathscr{Z}(M)$ (in the sense explained in section 1 and in 4.3). Since $N$ is finite, there exists a partition of the unity $\left\{p_{i}\right\}_{i \in I}$ in the center of $N$ such that $N_{p_{i}}$ is countable decomposable for each $i$. By $\S 3$ there exists an element $K_{0} \in \mathscr{J}(M)$ such that $\left(\delta-\operatorname{Ad} K_{0}\right)\left(p_{i}\right)$ $=0$ for all $i$. Thus we may assume that $\delta$ vanishes on $\left\{p_{i}\right\}_{i \in I}$. Moreover, by refining $\left\{p_{i}\right\}_{i}$ if necessary, we may assume $N_{p_{i}}$ is homogeneous for each $i$.

The unitary group of $N$ has an amenable subgroup $\mathscr{U}_{0}$ such that $\mathscr{U}_{0}^{\prime \prime}=\mathscr{Z}(N)$. Let $T_{0}=?_{\mathfrak{u}_{0}} \delta(u) u^{*} \mathrm{~d} \mu_{0}(u)$, where $\mu_{0}$ is the invariant mean on $\mathscr{U}_{0}$ and the integral has the usual significance (see e.g., [2]). Then $T_{0}$ is in the $\mathrm{K}_{\delta}$ set corresponding to $\mathscr{Z}(N)$, and by the same computations as in 2.12 we have

$$
T_{0} u_{0}-u_{0} T_{0}=\delta\left(u_{0}\right) \quad \text { for any } u_{0} \in \mathscr{U}_{0}
$$

Since both $\delta$ and ad $T_{0}$ are weakly continuous and $\mathscr{Z}(N)$ is the closed linear span of $\mathscr{U}_{0}$, it follows that $\delta=\operatorname{ad} T_{0}$ on $\mathscr{Z}(N)$ and thus 4.3 applies to get that $T_{0} \in \mathscr{J}(M)$. Thus, by taking $\delta-\operatorname{ad} T_{0}$ instead of $\delta$ if necessary, we may assume $\delta$ vanishes on $\mathscr{Z}(N)$.

Since $N_{p_{i}}$ is homogeneous, we have $N_{p_{i}} \simeq M_{n(i)} \otimes \mathscr{Z}_{i}$ for some $n(i) \times n(i)$ matrix algebra $M_{n(i)}$ and an abelian algebra $\mathscr{Z}_{i}$. Let $\mathscr{U}_{i}$ be the unitary group of $M_{n(i)} \otimes 1$ and $\mathscr{U}=\oplus \mathscr{U}_{i}$. Then $\mathscr{U}$ is amenable with an invariant mean $\mu$. Set $T=\int_{\mathscr{U}} \delta(u) u^{*} \mathrm{~d} \mu(u)$. Then $T$ is in the $\mathrm{K}_{\delta}$ set corresponding to $N$ and the integral $p_{i} \int_{\mathscr{U}_{i}} \delta(u) u^{*} \mathrm{~d} \mu(u) p_{i}=p_{i} \int_{\mathscr{U}^{\prime}} \delta(u) u^{*} \mathrm{~d} \mu(u) p_{i}$ is norm convergent (since $\mathscr{U}_{i}$ is compact). Thus $p_{i} T p_{i} \in \mathscr{J}(M)$ (as a norm limit of elements $\delta(u) u^{*}$ 
which are in $\mathscr{J}(M))$. Moreover, ad $T$ equals $\delta$ on $\mathscr{U}$ and also on $\mathscr{Z}(N)$. But $\mathscr{U}$ and $\mathscr{Z}(N)$ generate $N$, so that ad $T=\delta$ on $N$. Now if $T \notin \mathscr{J}(M)$ and, $\mathscr{P}=\left\{e \in \mathscr{P}(N)\left|\left\|\left|\|T e\|_{\text {ess }}=\|T \mid\|_{\text {ess }}\right\}\right.\right.\right.$, then it follows that $\mathscr{P}$ has no minimal projections (if $e$ would be such a minimal projection, then $e p_{i} \neq 0$ for some $i$ and $e-e p_{i}$ contradicts the minimality of $e$ ). To get a contradiction from this, we continue exactly as in the proofs of 4.3 or 4.5 . Thus $T$ lies in $\mathscr{J}(M)$ and the proof of Theorem 1.1 in the case where $N$ is finite type $\mathrm{I}$ is completed.

Assume now that $N$ is properly infinite. Then $N$ and $M$ are isomorphic to $N_{1} \bar{\otimes} \mathscr{B}\left(1^{2}(\mathbb{Z})\right)$ and $M_{1} \otimes \mathscr{B}\left(1^{2}(\mathbb{Z})\right)$, respectively, where $N_{1} \subset M_{1}$ are von Neumann algebras, in such a way that the inclusion $N \subset M$ becomes $N_{1} \bar{\otimes} \mathscr{B}\left(1^{2}(\mathbb{Z})\right) \subset M_{1} \bar{\otimes} \mathscr{B}\left(1^{2}(\mathbb{Z})\right)$. Note first that if the derivation $\delta: N \mapsto \mathscr{J}(M)$ vanishes on $\mathbb{C} 1 \bar{\otimes} \mathscr{B}\left(1^{2}(\mathbb{Z})\right) \subset N=N_{1} \otimes \mathscr{B}\left(1^{2}(\mathbb{Z})\right)$, then, given a unitary $u \in N_{1}$ $\otimes \mathbb{C} 1$, we have for any $x \in \mathbb{C} 1_{M_{1}} \otimes \mathscr{B}\left(1^{2}(\mathbb{Z})\right)$,

$$
\delta(u) x=\delta(u x)=\delta(x u)=x \delta(u)
$$

so that $\delta(u) \in \mathscr{J}(M) \cap\left(\mathbb{C} 1 \otimes \mathscr{B}\left(1^{2}(\mathbb{Z})\right)^{\prime} \cap M_{1} \bar{\otimes} \mathscr{B}\left(1^{2}(\mathbb{Z})\right)=J(M) \cap\left(M_{1} \otimes\right.\right.$ $\left.\mathbb{C} 1_{\mathscr{D}\left(1^{2}(\mathbf{Z})\right)}\right)=0$. Thus $\delta=0$ on $N$.

From this it follows that to prove the properly infinite case it is sufficient to prove the case when $\delta: N=\mathscr{B}\left(1^{2}(\mathbb{Z})\right) \mapsto \mathscr{J}(M)$.

Let $D$ be the diagonal von Neumann subalgebra of $\mathscr{B}\left(1^{2}(\mathbb{Z})\right)$ and $\mathrm{L}$ the von Neumann algebra generated by the bilateral shift $u$. Let $\sigma(x)=u x u^{*}$ for $x \in D$ be the automorphism of $D$ implemented by the shift $u$. By $\$ 3$ we may assume $\delta$ vanishes on $D$. Then for any $x \in D$ we have

$$
\begin{aligned}
x \delta\left(u^{n}\right) u^{-n} & =\delta\left(x u^{n}\right) u^{-n}=\delta\left(u^{n} \sigma^{-n}(x)\right) u^{-n} \\
& =\delta\left(u^{n}\right) \sigma^{-n}(x) u^{-n}=\delta\left(u^{n}\right) u^{-n} \sigma^{n}\left(\sigma^{-n}(x)\right)=\delta\left(u^{n}\right) u^{-n} x,
\end{aligned}
$$

which shows that $\delta\left(u^{n}\right) u^{-n} \in D^{\prime} \cap M$ for all $n \in \mathbb{Z}$.

But if we take $T$ to be a (weak) mean (after $n$ ) of $\delta\left(u^{n}\right) u^{-n}$, then $T \in D^{\prime} \cap M$ and, as in the preceding proof of the type I case, we have

$$
\left.\delta\right|_{L}=\left.\operatorname{ad} T\right|_{L}
$$

Thus ad $T$ equals $\delta$ on both $D$ and $L$. Since $\delta$ and ad $T$ are weakly continuous derivations, it follows that $\delta=\operatorname{ad} T$ on the von Neumann algebra generated by $D$ and $L$, which is easily seen to be $\mathscr{B}\left(1^{2}(\mathbb{Z})\right)=N$. Since $T$ belongs to the $\mathrm{K}_{\delta}$ set corresponding to $L, 4.5$ applies to get that $T \in \mathscr{J}(M)$.

6. Some technical results. To prove the remaining type $\mathrm{II}_{1}$ case of the theorem we need some technical devices that we prove below. As before, we 
continue to assume that $M$ is countable decomposable and use the notations of section 1.

6.1. Lemma. Let $N$ be a von Neumann algebra without atoms, $\psi$ a normal faithful state on $N$ and $\left\{w_{n}\right\}_{n}$ a sequence of unitary elements in $N$ such that $\psi\left(w_{n}^{k}\right) \rightarrow_{n} 0$ for all $k \neq 0$. Then there exist unitary elements $\left\{v_{n}\right\}_{n}$ in $N$ such that $\psi\left(v_{n}^{k}\right)=0, k \neq 0$, and $\left\|w_{n}-v_{n}\right\| \rightarrow 0$.

Proof. The proof is the same as the proof of 1.3 in [7], but we give it here anyway for the sake of completeness.

Since $N$ has no atoms, each $w_{n}$ is contained in some diffuse abelian von Neumann subalgebra $A_{n} \subset N$ with separable predual and $\left(A_{n},\left.\psi\right|_{A_{n}}\right)$ can be identified by some measure preserving isomorphism $\varphi_{n}$ with $L^{\infty}(\mathbb{T}, \mu)$, where $\mu$ is the normalized Lebesgue measure on the torus T. Moreover, $\varphi_{n}$ can be chosen so that $\varphi_{n}\left(w_{n}\right)=f_{n}$, where $f_{n}\left(e^{2 \pi i t}\right)=e^{2 \pi i h_{n}(t)}$ for some nondecreasing function $h_{n}:[0,1] \mapsto[0,1]$. By Helly's selection principle there exists a subsequence $\left\{h_{k_{n}}\right\}_{n}$ tending everywhere to some nondecreasing function $h:[0,1] \rightarrow[0,1]$. Thus, if $f\left(e^{2 \pi i t}\right)=e^{2 \pi i h(t)}$, then $\left\{f_{k_{n}}\right\}_{n}$ tends everywhere to $f$, so that by Lebesgue's theorem $\int f_{k_{n}} \mathrm{~d} \mu \mapsto \int f^{p} \mathrm{~d} \mu$ for all $p$, which by the hypothesis implies $\int f^{p} \mathrm{~d} \mu=0$ for $p \neq 0$. Thus $\int q(f) \mathrm{d} \mu=\int q \mathrm{~d} \mu$ for Laurent polynomials $q$ so that $\int g \circ f \mathrm{~d} \mu$ $=\int g \mathrm{~d} \mu$ for any $g \in L^{\infty}(\mathbb{T}, \mu)$. In particular, if we define $g_{z}\left(\mathrm{e}^{2 \pi i s}\right)=\left\{\begin{array}{l}1 \text { if } 0 \leqslant s<t \\ 0 \text { if } t \leqslant s<1\end{array}\right.$, where $z=e^{2 \pi i t}$, then we get $\int_{h(s) \leqslant t} \mathrm{~d} \lambda(s)=\int g_{z} \circ f \mathrm{~d} \mu=\int g_{z} \mathrm{~d} \mu=t, \lambda$ being the Lebesgue measure on $[0,1]$. This implies $h(t)=t$ and hence $f(z)=z$ is the identity function on $\pi$. Now, since $h_{k_{n}}$ are monotone and converge everywhere to a continuous function, it follows that $h_{k_{n}}$ converge uniformly to $h$, so that $\left\|f_{k_{n}}-f\right\| \rightarrow 0$. Since any limit point of $f_{k_{n}}$ was shown to be equal to the identity $f$, it follows that $\left\|f_{n}-f\right\| \rightarrow 0$.

We can now take $v_{n}=\varphi_{n}^{-1}(f)$. Since $\int f^{p} \mathrm{~d} \mu=0, \psi\left(v_{n}^{p}\right)=0$ for all $p \neq 0$. Moreover, $\left\|w_{n}-v_{n}\right\|=\left\|\varphi_{n}\left(w_{n}\right)-\varphi_{n}\left(v_{n}\right)\right\|=\left\|f-f_{n}\right\| \rightarrow 0$. $\quad$ Q.E.D.

6.2. Lemma. (1). Let $N \subset M$ be a von Neumann subalgebra such that $N^{\prime} \cap M$ contains no finite projections of $M$. Let $\varepsilon>0$ and $e, f$ two finite projections of $M$ with $\varphi(e)<\infty, \varphi(f)<\infty$. Then there exists a unitary element $u \in N$ such that $\|$ fue $\|_{\varphi}<\varepsilon$. Moreover, if $N$ is abelian, then, given any $n \geqslant 1$, there exists a unitary element $u \in N$ such that $\left\|f u^{k} e\right\|_{\varphi}<\varepsilon$ for $k \neq 0,|k| \leqslant n$.

(2). If $N$ is of type $\mathrm{II}_{1}$ and countable decomposable, $M$ is countable decomposable, and $N^{\prime} \cap M$ contains no finite projections of $M$, then there exist an approximately finite-dimensional type $\mathrm{II}_{1}$ von Neumann algebra $R \subset N$ which contains a diffuse abelian von Neumann subalgebra $A \subset R$ such that $A^{\prime} \cap M$ contains no finite projections of $M$. Moreover, if $N$ has separable predual, then we can make the construction so that, in addition to the above properties, $A$ is maximal abelian in $N$.

Proof. (1). Let $\varphi_{n}$ be the semifinite faithful trace on $M^{2 n}$ given by $\varphi_{n}\left(\left(x_{k}\right)_{|k| \leqslant n, k \neq 0}\right)=\sum \varphi\left(x_{k}\right)$. Set $\mathrm{K}_{e}^{n}=\overline{\operatorname{co}}^{\mathrm{w}}\left\{\left(u^{k} e u^{-k}\right)_{|k| \leqslant n, k \neq 0} \mid u\right.$ unitary element of $N\} \subset M^{2 n}$. Then $\varphi_{n}(\bar{x}) \leqslant 2 n \varphi(e)$ and $\|\bar{x}\| \varphi_{n} \leqslant 2 n\|e\|_{\varphi}$ for any $\bar{x} \in \mathrm{K}_{e}^{n}$. 
By the inferior semicontinuity of the norm \|\|$_{\varphi_{n}}$, there exists a unique element $\bar{x}_{0} \in \mathrm{K}_{e}^{n}$ with $\left\|\bar{x}_{0}\right\|_{\varphi_{n}} \leqslant\|\bar{x}\|_{\varphi_{n}}$ for all $x \in \mathrm{K}_{e}^{n}$. But if $N$ is abelian, then for any unitary element $u \in N$, if $\tilde{u}=\left(u^{k}\right)_{|k| \leqslant n, k \neq 0}$, then $\tilde{u} \mathrm{~K}_{e}^{n} \tilde{u}^{*} \subset \mathrm{K}_{e}^{n}$ and $\left\|\tilde{u} \bar{x}_{0} \tilde{u}^{*}\right\|_{\varphi_{n}}$ $=\left\|x_{0}\right\|_{\varphi_{n}}$ so that, by the uniqueness of $\bar{x}_{0}, \tilde{u} \bar{x}_{0} \tilde{u}^{*}=\bar{x}_{0}$. Thus if $\bar{x}_{0}=$ $\left(x_{k}\right)_{|k| \leqslant n, k \neq 0} \neq 0$, then $x_{k} \neq 0$ for some $k$ and $u^{k} x_{k}=x_{k} u^{k}$ for any unitary element $u \in N$. Since in a von Neumann algebra $N$ any unitary element $v \in N$ can be written as $u^{k}$ for some $u \in N$, it follows that $v x_{k}=x_{k} v$ for unitary elements $v \in N$, and by taking linear combinations, $y x_{k}=x_{k} y$ for all $y \in N$. But $0<\left\|x_{k}\right\|_{\varphi} \leqslant\|e\|_{\varphi}$ and $x_{k} \in N^{\prime} \cap M$, a contradiction. This shows that $0=x_{0} \in \mathrm{K}_{e}^{n}$, so that given any $\varepsilon>0$ and any $f \in\left(M_{\varphi}\right)_{+}$there is a $u \in \mathscr{U}(N)$ such that $\sum_{0<|k| \leqslant n} \varphi\left(f u^{k} e u^{-k}\right)<\varepsilon^{2}$. Thus $\left\|f u^{k} e\right\|_{\varphi}<\varepsilon$ for all $k \neq 0,|k| \leqslant n$. If $N$ is arbitrary, we take $M$ instead of $M^{2 n}$ and the proof is the same.

(2). The argument we use is similar to the one in [6]. We first prove that if $p \in N$, then $N_{p}^{\prime} \cap M_{p}$ contains no finite projections of $M_{p}$. To show this, let $f \neq 0$ be a projection in $N_{p}^{\prime} \cap M_{p}$ and $z$ a projection in the center of $N$. Then $z f \in N_{p}^{\prime} \cap M_{p}$, and if $f$ is finite in $M_{p}$, then $z f$ is finite in $M_{z p}$. Take $z$ to be so that $f z \neq 0$ and $p z$ divides $z$, say $n$ times. It follows that the inclusion $N_{z} \subset M_{z}$ is the same as $N_{z p} \otimes M_{n \times n} \subset M_{z p} \otimes M_{n \times n}$ and that $f^{\prime}=z f \otimes I_{n} \in$ $\left(N_{z p} \otimes M_{n \times n}\right)^{\prime} \cap\left(M_{z p} \otimes M_{n \times n}\right)$. Hence $f^{\prime} \in N_{z}^{\prime} \cap M_{z}=z\left(N^{\prime} \cap M\right) z \subset N^{\prime} \cap$ $M$, and if $f$ is finite, then $f^{\prime}$ is finite, contradicting the hypothesis.

Since $M$ is countable decomposable, there exists an increasing sequence of finite projections $\left\{f_{n}\right\}_{n}$ in $M$ with $f_{n} \uparrow 1$. Moreover, by cutting each $f_{n}$ with a central projection if necessary, we may assume $\varphi\left(f_{n}\right)<\infty, n \in \mathbb{N}$.

We now recursively construct an increasing sequence of finite-dimensional von Neumann subalgebras $R_{k}$ in $N$ with matrix units $\left\{e_{i j}^{k p}\right\}_{1 \leqslant i, j \leqslant n(k, p)}$ satisfying the following properties:

1. Each $e_{s t}^{k-1, r}$ is the sum of some $e_{i j}^{k p}$.

2. If $A_{k}$ is the diagonal algebra of $R_{k}$ generated by $\left\{e_{i i}^{k p}\right\}_{i, p}$, then $\left\|E_{A_{k}^{\prime} \cap M}\left(f_{k}\right)\right\|_{\varphi}^{2}<(3 / 4)^{k}$.

3. $n(k, p) \geqslant k$ for each $p=1,2, \ldots, m(k)$.

Assume we have constructed these objects up to some $k$. By (1) it follows that for each $g=e_{11}^{k p}$ there exists a unitary element $u \in N_{g}$ such that if $e$ is the support of $\sum_{i} e_{1 i}^{k p} f_{k+1} e_{i 1}^{k p}$, then for each nonzero $x_{i}=e_{1 i}^{k p} f_{k+1} e_{i 1}^{k p}$ we have $\varphi\left(\right.$ eueu $\left.^{*}\right)=\|e u e\|_{\varphi}^{2}<1 / 2\left\|x_{i}\right\|_{\varphi}^{2}$. Approximating $u$ in the uniform norm, we may assume it has finite spectrum so that $u=\sum \lambda_{s} e_{s}$ with $\sum e_{s}=g$ and $\left|\lambda_{s}\right|=1$. Then, since $\varphi\left(x_{i} u x_{i} u^{*}\right) \leqslant \varphi\left(e u e u^{*}\right)$, we have

$$
\begin{aligned}
\left\|x_{i}\right\|_{\varphi}^{2} & =2\left\|x_{i}\right\|_{\varphi}^{2}-\left\|x_{i}\right\|_{\varphi}^{2} \leqslant\left\|x_{i}\right\|_{\varphi}^{2}+\left\|u x_{i} u^{*}\right\|_{\varphi}^{2}-2 \varphi\left(x_{i} u x_{i} u^{*}\right) \\
& =\left\|x_{i}-u x_{i} u^{*}\right\|_{\varphi}^{2}=\left\|\sum_{r \neq s}\left(\lambda_{r} \bar{\lambda}_{s}-1\right) e_{r} x_{i} e_{s}\right\|_{\varphi}^{2} \\
& \leqslant 4 \sum_{r \neq s}\left\|e_{r} x_{i} e_{s}\right\|_{\varphi}^{2}=4\left\|x_{i}\right\|_{\varphi}^{2}-4 \sum_{r}\left\|e_{r} x_{i} e_{r}\right\|_{\varphi}^{2} .
\end{aligned}
$$


Thus $\sum_{r}\left\|e_{r} x_{i} e_{r}\right\|_{\varphi}^{2} \leqslant 3 / 4\left\|x_{i}\right\|_{\varphi}^{2}$. Now we can apply the same trick to $e_{r} x_{i} e_{r}$ instead of $x_{i}$ and get a refinement $\left\{e_{s}^{2}\right\}_{s}$ of the projections $e_{r}^{1}=e_{r}$, so that $\left\|\sum_{s} e_{s}^{2} x_{i} e_{s}^{2}\right\|_{\varphi}^{2} \leqslant(3 / 4)^{2} \sum\left\|e_{r}^{1} x_{i} e_{r}^{1}\right\|_{\varphi}^{2} \leqslant(3 / 4)^{2}\left\|x_{i}\right\|_{\varphi}^{2}$. More generally, we apply the trick $k+1$ times to get projections $g_{l}=e_{l}^{k+1}$ so that $\left\|\Sigma_{l} g_{l} x_{i} g_{l}\right\|_{\varphi}^{2} \leqslant$ $(3 / 4)^{k+1}\left\|x_{i}\right\|_{\varphi}^{2}$ and $\Sigma_{l} g_{l}=e_{11}^{k p}$.

Now since $N$ is type $\mathrm{II}_{1}$, each $g_{l}$ can be divided into $k+1$ mutually orthogonal equivalent projections. Thus we may consider matrix units $\left\{g_{a b}^{l}\right\}_{1 \leqslant a, b \leqslant k+1}$ with $\sum_{a} g_{a a}^{l}=g_{l}$. Then easy computations show that if we denote by $\left\{e_{s t}^{k+1, r}\right\}_{s, t, r}$ an appropriate relabeling of $\left\{e_{i 1}^{k p} g_{a b}^{l} e_{1 j}^{k p}\right\}_{a, b, l, i, j, p}$, then this matrix unit and the von Neumann algebra $R_{k+1}$ generated by it, together with its diagonal $A_{k+1}$, will satisfy conditions 1,2 , and 3 .

Let $R={\overline{\bigcup_{k} R}}_{k}^{w}$. Then condition 3 implies that $R$ is of type $\mathrm{II}_{1}$.

Let $A={\overline{U_{n} A}}_{n}^{w}$. Suppose $e \in A^{\prime} \cap M, e \neq 0$, is a finite projection of $M$. Then by cutting $e$ with a projection in $\mathscr{Z}(M)$ if necessary, we may assume $\varphi(e)<\infty$. Since $f_{n} \uparrow 1$, there exists $n$ such that $\left\|f_{n} e f_{n}-e\right\|_{\varphi}<1 / 2\|e\|_{\varphi}$. By the construction of $A_{n} \subset A$ there exists a partition of the unity $e_{1}, \ldots, e_{m}$ with projections in $A$ such that

$$
\left\|\sum_{i} e_{i} f_{n} e_{i}\right\|_{\varphi}<1 / 2\|e\|_{\varphi}
$$

But then

$$
\left\|\sum_{i} e_{i} f_{n} e f_{n} e_{i}\right\|_{\varphi} \leqslant\left\|\sum_{i} e_{i} f_{n} e_{i}\right\|_{\varphi}<1 / 2\|e\|_{\varphi}
$$

so that, since $e=\sum_{i} e_{i} e e_{i}$,

$$
\|e\|_{\varphi}=\left\|\sum_{i} e_{i} e e_{i}\right\|_{\varphi}<\left\|\sum_{i} e_{i}\left(f_{n} e f_{n}-e\right) e_{i}\right\|_{\varphi}+\left\|\sum_{i} e_{i} f_{n} e f_{n} e_{i}\right\|_{\varphi}<\|e\|_{\varphi}
$$

which is a contradiction.

Finally, if we assume $N$ is separable, then it has a normal faithful trace $\tau$ and there is a set of elements $\left\{y_{k}\right\}_{k} \subset N$ dense in $N$ in the norm $\|y\|_{2}=\tau\left(y^{*} y\right)^{1 / 2}$ and we may construct recursively $A_{k}, R_{k}$, so that, in addition to conditions 1,2 , and 3 , to satisfy condition $4,\left\|E_{A_{k}^{\prime} \cap M}\left(y_{i}\right)-E_{A_{k}}\left(y_{i}\right)\right\|_{2}<1 / k, 1 \leqslant i \leqslant k$.

Then by [6] it follows that, besides the above properties, $A$ is also maximal abelian in $N$.

Q.E.D.

In the rest of this section $N \subset M$ will be a type $\mathrm{II}_{1}$ von Neumann subalgebra with a fixed normal finite faithful trace $\tau, \tau(1)=1$. The norm on $N$ given by $\tau$ is 
denoted $\|x\|_{2}=\tau\left(x^{*} x\right)^{1 / 2}, x \in M$. If $B \subset N$ is a von Neumann subalgebra, then $E_{B}$ denotes the unique normal $\tau$-preserving conditional expectation onto $B$ (cf. [10]).

6.3. Lemma. Assume $A \subset N$ is a maximal abelian von Neumann subalgebra of $N$ such that $A^{\prime} \cap M$ contains no finite projections of $M$. Let $\varepsilon>0, n \geqslant 1$, e and $f$ finite projections in $M_{\varphi}$ and $v$ a unitary element in $N$. Then there exists a unitary element $u \in A$ such that $\left\|f(u v)^{k} e\right\|_{\varphi}^{2} \leqslant \varepsilon$ for any $k \neq 0,|k| \leqslant n$.

Proof. Since $\varphi(e), \varphi(f)<\infty$, it follows that $\varphi(e \vee f)<\infty$. Since $\|(e \vee f)$ $(u v)^{k}(e \vee f)\left\|_{\varphi} \geqslant\right\| f(u v)^{k} e \|_{\varphi}$, it is sufficient to prove the statement when $e=f$. Since $\left\|e(u v)^{k} e\right\|_{\varphi}=\left\|e(u v)^{-k} e\right\|_{\varphi}$, we only need to prove the estimates for $k>0$. We'll actually prove the following more general result:

(*) If $\varepsilon>0, n \geqslant 1, \mathscr{F} \subset N$ is a finite self-adjoint set of norm one elements containing the identity and $e, f$ are finite projections in $M_{\varphi}$, then there exists a unitary element $u \in A$ such that

$$
\left\|f x_{0} \prod_{i=1}^{k}\left(u x_{i}\right) e\right\|_{\varphi}^{2}<\varepsilon
$$

for any $1 \leqslant k \leqslant n$ and $x_{0}, x_{1}, \ldots, x_{k} \in \mathscr{F}$.

We first prove $(*)$ in the case $\varphi(x e) \leqslant c \tau(x), \varphi(f x) \leqslant c \tau(x), x \in N_{+}$, for some constant $c>0$. Let $\mathscr{W}=\left\{w\right.$ partial isometry in $A \mid\left\|f x_{0} \Pi_{i=1}^{k}\left(w x_{i}\right) e\right\|_{\varphi}^{2} \leqslant$ $\varepsilon \tau\left(w^{*} w\right)$ for any $\left.1 \leqslant k \leqslant n, x_{0}, x_{1}, \ldots, x_{k} \in \mathscr{F}\right\}$ and consider on $\mathscr{W}$ the usual order: $w_{0} \leqslant w_{1}$ if $w_{0}$ is a restriction of $w_{1}$, i.e., $w_{0}=w_{1} w_{0}^{*} w_{0}$. The set is clearly inductively ordered. Let $u$ be a maximal element of it and suppose $u^{*} u \neq 1$. Denote by $A_{0}=\left(1-u^{*} u\right) A\left(1-u^{*} u\right), N_{0}=\left(1-u^{*} u\right) N\left(1-u^{*} u\right)$, and $\mathscr{F}_{0}=$ $\left\{\left(1-u^{*} u\right) x_{0}\left(\Pi_{i=1}^{k}\left(u x_{i}\right)\right)\left(1-u^{*} u\right) \mid 1 \leqslant k \leqslant n, x_{0}, x_{1}, \ldots, x_{n} \in \mathscr{F}\right\}$. By 1.2 in [6], given any $\delta>0$, there exists a partition of the unity $e_{1}, \ldots, e_{m}$ in $A_{0}$ such that $\sum_{i}\left\|e_{i} y e_{i}-E_{A_{0}}(y) e_{i}\right\|_{2}^{2}=\left\|\sum_{i} e_{i} y e_{i}-E_{A}(y)\right\|_{2}^{2}<\delta \tau\left(1-u^{*} u\right)=\delta \sum_{i} \tau\left(e_{i}\right)$ for all $y \in \mathscr{F}_{0}$. It follows that for some $e_{0}=e_{i}$ we have

$$
(* *) \quad\left\|e_{0} y e_{0}-E_{A_{0}}(y) e_{0}\right\|_{2}^{2}<\delta \tau\left(e_{0}\right), \quad y \in \mathscr{F}_{0} .
$$

Let $n \geqslant r, s \geqslant 0, x \in \mathscr{F}, y, \quad y_{1}, \ldots, y_{s} \in \mathscr{F}_{0}, x^{\prime} \in \mathscr{F}^{*}, y^{\prime}, \quad y_{1}^{\prime}, \ldots, y_{r}^{\prime} \in \mathscr{F}_{0}$ and $w \in A_{0} e_{0},\|w\| \leqslant 1$ and put $\alpha=\left|\varphi\left(e x^{\prime} \Pi_{i=1}^{r}\left(y_{i}^{\prime} w^{*}\right) y^{\prime} f y \Pi_{j=1}^{s}\left(w y_{j}\right) x e\right)\right|$, with the convention that a product over a void set equals 1 .

If $s=1$, then by the Cauchy-Schwartz inequality we have

$$
\begin{aligned}
\alpha & \leqslant\left\|f y w y_{1} x e\right\|_{\varphi}\left\|e x^{\prime}\left(\prod_{i=1}^{r} y_{i}^{\prime} w^{*}\right) y^{\prime} f\right\|_{\varphi} \leqslant\left\|f y w v_{1} x e\right\|_{\varphi}\|e\|_{\varphi} \\
& \leqslant\|\bar{f} w \bar{e}\|_{\varphi}\|e\|_{\varphi}
\end{aligned}
$$


where $\bar{e}$ is the supremum of the left supports of all the elements of the form $z y_{1} x e$, with $x \in \mathscr{F}, \quad y_{1} \in \mathscr{F}_{0}$, and $z \in \mathscr{F}_{1}=\left\{\Pi_{i=1}^{k} E_{A}\left(y_{i}\right) e_{0} \mid 0 \leqslant k \leqslant n\right.$, $\left.y_{1}, \ldots, y_{k} \in \mathscr{F}_{0}\right\}$, and $\bar{f}$ is the supremum of the right supports of all the elements fy with $y \in \mathscr{F}_{0}$.

If $s \geqslant 2$, then we have

$$
\begin{aligned}
&\left\|\bar{f} \prod_{i=1}^{s}\left(w y_{i}\right) x e\right\|_{\varphi} \leqslant \sum_{j=1}^{s-1} \| \prod_{i=1}^{j-1}\left(w y_{i}\right) w\left(E_{A}\left(y_{j}\right) e_{0}-e_{0} y_{j} e_{0}\right) \\
& w^{s-j}\left(\prod_{t=j+1}^{s-1} E_{A}\left(y_{t}\right)\right) y_{s} x e\left\|_{\varphi}+\right\| \bar{f} w^{s} \prod_{j=1}^{s-1} E_{A}\left(y_{j}\right) y_{s} x e \|_{\varphi} \\
& \leqslant \sum\left\{\left\|\left(E_{A}\left(y_{0}\right) e_{0}-e_{0} y_{0} e_{0}\right) w^{j} z y x e\right\|_{\varphi} \mid 1 \leqslant j \leqslant s,\right. \\
&\left.x \in \mathscr{F}, y_{0}, y \in \mathscr{F}_{0}, z \in \mathscr{F}_{1}\right\}+\left\|\bar{f} w^{s} \bar{e}\right\|_{\varphi},
\end{aligned}
$$

where $\bar{e}, \bar{f}$ are as before. Thus if $\beta$ denotes the sum in the right-hand side of the above last inequality, then by $(* *)$ we get $\beta \leqslant s N N_{0}^{2} N_{1} c^{1 / 2} \delta^{1 / 2}\left\|e_{0}\right\|_{2}$, where $N$, $N_{0}$, and $N_{1}$ are the number of elements in $\mathscr{F}, \mathscr{F}_{0}$, and $\mathscr{F}_{1}$, respectively.

Thus, by the Cauchy-Schwartz inequality we obtain

$$
\begin{aligned}
\alpha & \leqslant\left\|e x^{\prime} \prod_{i=1}^{r}\left(y_{i}^{\prime} w^{*}\right) y^{\prime} f y e_{0}\right\|_{\varphi}\left(\beta+\left\|\bar{f} w^{s} \bar{e}\right\|_{\varphi}\right) \\
& \leqslant\left\|f y e_{0}\right\|_{\varphi}\left(\beta+\left\|\bar{f} w^{s} \bar{e}\right\|_{\varphi}\right) \leqslant c^{1 / 2}\left\|e_{0}\right\|_{2}\left(\beta+\left\|\bar{f} w^{s} \bar{e}\right\|_{\varphi}\right) \\
& \leqslant s N N_{0}^{2} N_{1} c \delta^{1 / 2}\left\|e_{0}\right\|_{2}^{2}+c^{1 / 2}\left\|e_{0}\right\|_{2}\left\|\bar{f} w^{s} \bar{e}\right\|_{\varphi} .
\end{aligned}
$$

Thus, if $\delta$ is such that $\operatorname{sn} N N_{0}^{2} N_{1} c \delta^{1 / 2}<\varepsilon 2^{-2 n-1}$ and if, using 6.2, we choose $w$ to be a unitary element in $A_{0} e_{0}=A e_{0} \subset e_{0} M e_{0}$ such that $c^{1 / 2}\left\|\bar{f} w^{s} \bar{e}\right\|_{\varphi}<$ $\varepsilon 2^{-2 n-1}\left\|e_{0}\right\|_{2}$, then we get $\alpha<2^{-2 n} \varepsilon \tau\left(e_{0}\right)$.

We now show that if $w$ is chosen like this, then $u_{0}=u+w$ contradicts the maximality of $u$. Indeed we have for any $1 \leqslant k \leqslant n$ and $x_{0}, x_{1}, \ldots, x_{k} \in \mathscr{F}$ :

$$
\left\|f x_{0}\left(\prod_{i=1}^{k}(u+w) x_{i}\right) e\right\|_{\varphi}^{2} \leqslant\left\|f x_{0} \prod_{i=1}^{k}\left(u x_{i}\right) e\right\|_{\varphi}^{2}+\sum \alpha,
$$


where the $\alpha$ 's appearing in the sum are of the form estimated above and there are $2^{k}-1$ terms in that sum. It follows that $\sum \alpha \leqslant \varepsilon \tau\left(e_{0}\right)$, so that

$$
\left\|f x_{0}\left(\prod_{i=1}^{k}(u+w) x_{i}\right) e\right\|_{\varphi}^{2} \leqslant \varepsilon\left(\tau\left(u^{*} u\right)+\tau\left(w^{*} w\right)\right)=\varepsilon \tau\left((u+w)^{*}(u+w)\right) .
$$

This ends the proof of $(*)$ in the case $\varphi(x e) \leqslant c \tau(x), \varphi(f x) \leqslant c \tau(x)$, for $x=N_{+}$.

To prove the general case, i.e., for arbitrary $e, f$ in $M_{\varphi}$, note that given any $\varepsilon>0$ there exist finite projections $e^{\prime}, f^{\prime} \in M_{\varphi}$ with $\left\|e-e^{\prime}\right\|_{\varphi}<\varepsilon / 3$, $\left\|f-f^{\prime}\right\|_{\varphi}<\varepsilon / 3$, and such that $\varphi\left(x e^{\prime}\right) \leqslant c \tau(x), \varphi\left(f^{\prime} x\right) \leqslant c \tau(x)$ for some constant $c>0$. Indeed, since $\varphi(. e), \varphi(f$.$) are in N_{*}$, there exist $X, Y \in L^{1}(N, \tau)_{+}$ such that $\varphi(x e)=\tau(x X), \varphi(f x)=\tau(x Y)$, for $x \in N$. Thus if $E_{n}, F_{n}$ are the spectral projections of $X$ and $Y$, respectively, corresponding to the intervals $[0, n]$, then $E_{n} \uparrow 1, \quad F_{n} \uparrow 1$ and $\varphi\left(x E_{n} e E_{n}\right)=\varphi\left(E_{n} x E_{n} e\right)=\tau\left(E_{n} x E_{n} X\right)=$ $\tau\left(x E_{n} X\right) \leqslant n \tau(x)$ and, similarly, $\varphi\left(F_{n} f F_{n} x\right) \leqslant n \tau(x)$. It follows that $\| E_{n} e E_{n}-$ $e\left\|_{\varphi} \mapsto 0,\right\| F_{n} f F_{n}-f \|_{\varphi} \mapsto 0$, so that if $e_{n}^{\prime}, f_{n}^{\prime}$ are the spectral projections of $E_{n} e E_{n}$ and $F_{n} f F_{n}$, respectively, corresponding to the interval $[1 / 2, \infty)$, then any easy computation shows that $\left\|e_{n}^{\prime}-e\right\|_{\varphi} \mapsto 0,\left\|f_{n}^{\prime}-f\right\|_{\varphi} \mapsto 0$ and $\varphi\left(x e_{n}^{\prime}\right) \leqslant$ $2 \varphi\left(x E_{n} e E_{n}\right) \leqslant 2 n \tau(x), \varphi\left(f_{n}^{\prime} x\right) \leqslant 2 \varphi\left(F_{n} f F_{n} x\right) \leqslant 2 n \tau(x)$ (see, e.g., 1.4 in [8]). Now by the first part of the proof, given $\varepsilon>0$ and $n \geqslant 1$, there exists a unitary element $u \in A$ such that $\left\|f^{\prime} x_{0} \Pi_{i=1}^{k}\left(u x_{i}\right) e^{\prime}\right\|_{\varphi} \leqslant \varepsilon / 3$ for any $1 \leqslant k \leqslant$ $n, x_{0}, x_{1}, \ldots, x_{k} \in \mathscr{F}$. But then

$$
\left\|f x_{0} \prod_{i=1}^{k}\left(u x_{i}\right) e\right\|_{\varphi}<2 \varepsilon / 3+\left\|f^{\prime} x_{0} \prod_{i=1}^{k}\left(u x_{i}\right) e^{\prime}\right\|_{\varphi} \leqslant 2 \varepsilon / 3+\varepsilon / 3=\varepsilon \text {. Q.E.D. }
$$

6.4. Corollary. Let $\varepsilon>0, n \geqslant 1, e, f$ be two finite projections in $M_{\varphi}$ and $v \in N$ a unitary element. There exist a finite projection $e_{n} \in M$ and a unitary element $w \in N$ such that

1. $\varphi\left(e_{n} w^{k} e_{n}\right)=0$ for any $k \neq 0$;

2. $e_{n} \leqslant e, \varphi\left(e-e_{n}\right)<\varepsilon$;

3. $\left\|f w^{k} e_{n}\right\|<\varepsilon$ for $k \neq 0,|k| \leqslant n$;

4. $\|w-u v\|<\varepsilon$ for some unitary element $u \in A$.

Proof. First we prove that given any $\varepsilon^{\prime}>0$ there exist unitary elements $u \in A$ and $w^{\prime} \in N$ and a finite projection $e_{n} \in M$ such that

(a) $e_{n} \leqslant e, \varphi\left(e-e_{n}\right)<\varepsilon^{\prime}$;

(b) $\left\|f w^{\prime k} e_{n}\right\|<\varepsilon^{\prime}$ for $k \neq 0,|k| \leqslant n$;

(c) $\left\|w^{\prime}-u v\right\|<\varepsilon^{\prime}$;

(d) $\varphi\left(e w^{\prime k} e\right)=0$ for all $k \neq 0$. 
Then it follows by (a) and (d) that $\left|\varphi\left(w^{\prime k} e_{n}\right)\right| \leqslant \varepsilon^{\prime}$ for any $k \neq 0$ and thus if $\varepsilon^{\prime}$ is small enough and $\varepsilon^{\prime} \leqslant \varepsilon / 2$, by 6.1 there exists a unitary element $w \in N$ such that $\left\|w-w^{\prime}\right\| \leqslant \varepsilon / 2 n$ and $\varphi\left(w^{k} e_{n}\right)=0$ for any $k \neq 0$. But then $\left\|f w^{k} e_{n}\right\| \leqslant$ $\left\|f w^{\prime} e_{n}\right\|+n\left\|w-w^{\prime}\right\|<\varepsilon$ for $k \neq 0, \quad|k| \leqslant n$, and $\|w-u v\| \leqslant\left\|w-w^{\prime}\right\|+$ $\left\|w^{\prime}-u v\right\| \leqslant \varepsilon / 2 n+\varepsilon / 2 \leqslant \varepsilon$.

Now to prove $(*)$ we let $\varepsilon^{\prime \prime}>0, n^{\prime} \geqslant 1$. By the preceding lemma there exists a unitary element $u \in A$ such that $\left\|(e \vee f)(u v)^{k} e\right\|_{\varphi}<\varepsilon^{\prime \prime}$ for $k \neq 0,|k| \leqslant n^{\prime}$. It follows that $\left|\varphi\left(e(u v)^{k} e\right)\right| \leqslant\|e\|_{\varphi}\left\|e(u v)^{k} e\right\|_{\varphi}<\varepsilon^{\prime \prime}\|e\|_{\varphi}$, for all $k \neq 0,|k| \leqslant n^{\prime}$, and $\varphi\left(e(u v)^{-k} f(u v)^{k} e\right)=\left\|f(u v)^{k} e\right\|_{\varphi}^{2}<\varepsilon^{\prime \prime 2}$. If $e_{k}^{\prime}$ is the spectral projection of $e(u v)^{-k} f(u v)^{k} e$ corresponding to the interval $\left(0, \varepsilon^{\prime \prime}\right]$, then $e_{k}^{\prime} \leqslant$ $e, e_{k}^{\prime}(u v)^{-k} f(u v)^{k} e_{k}^{\prime} \leqslant \varepsilon^{\prime \prime}$ and $e-e_{k}^{\prime} \leqslant \varepsilon^{\prime \prime-1} e(u v)^{-k} f(u v)^{k} e$ so that $\varphi\left(e-e_{k}^{\prime}\right)$ $\leqslant \varepsilon^{\prime \prime-1} \varepsilon^{\prime \prime 2}=\varepsilon^{\prime \prime}$. Let $e_{n}=\wedge\left\{e_{k}^{\prime}|k \neq 0| k \mid, \leqslant n\right\}$. Then $e_{n} \leqslant e, \varphi\left(e_{n}\right) \geqslant \varphi(e)-$ $2 n \varepsilon^{\prime \prime}$, and $\left\|f(u v)^{k} e_{n}\right\|^{2} \leqslant\left\|f(u v)^{k} e_{k}^{\prime}\right\|^{2} \leqslant \varepsilon^{\prime \prime}$.

Lemma 6.1 shows that if $n^{\prime}$ is large enough and $\varepsilon^{\prime \prime}$ is small enough with $\varepsilon^{\prime \prime}<\left(\varepsilon^{\prime} /(n+1)\right)^{2}$, then there exists a unitary element $w^{\prime} \in N$ such that $\varphi\left(w^{\prime k} e\right)=0$ for all $k \neq 0$ and $\left\|w^{\prime}-u v\right\|<\varepsilon^{\prime} / n+1$. But then $\left\|f w^{\prime k} e_{n}\right\| \leqslant \sum_{p=0}^{k-1}\left\|f(u v)^{p}\left(w^{\prime}-u v\right)(w)^{k-p-1} e_{n}\right\|+\left\|f(u v)^{k} e_{n}\right\| \leqslant k \varepsilon^{\prime} /(n+1)+$ $\varepsilon^{\prime} /(n+1)=(k+1) \varepsilon^{\prime} /(n+1) \leqslant \varepsilon^{\prime}$, which proves $(*)$.

Q.E.D.

7. End of the proof of Theorem 1.1: The type $\mathrm{II}_{1}$ case. In this section we prove 1.1 in the case where $N$ is of type $\mathrm{II}_{1}$. By 2.11 and $\S 5$ this will end the proof of the theorem. We begin the section by reducing the problem in several steps to the case when the type $\mathrm{II}_{1}$ von Neumann algebra $N$ is separable, $M$ is countable decomposable, and $N^{\prime} \cap M$ contains no finite projections of $M$. Note from the beginning that by section 3 we may assume $\delta$ vanishes on a set of projections $\left\{p_{i}\right\}_{i}$ in the center of $M$ having the properties $\Sigma p_{i}=1$ and $N_{p_{i}}$ is of countable type for each $i$.

7.1. First reduction. It is sufficient to prove the theorem for separable $N$ (i.e., $N$ with separable predual).

To show this, let $R \subset N$ be a copy of the hyperfinite type $\mathrm{II}_{1}$ factor with the same unit as $N$ (cf. [5]). There exists an increasing net of separable von Neumann subalgebras $\left\{N_{i}\right\}_{i}$ of $N$ with $R \subset N_{i}$ and $\bar{U}_{i} N_{i}^{w}=N$. Indeed, if $\left\{p_{j}\right\}_{j \in J}$ is a partition of the unity in the center of $N$ such that $N p_{j}$ is countable decomposable for each $j$, then any countably generated von Neumann subalgebra of $N p_{j}$ is separable, so that if $N_{i}$ are such that $N_{i} p_{j}$ is countably generated and contains $R p_{j}$ for a finite number $J_{0}$ of $j \in J$ and if $N_{i} \sum_{j \notin J_{0}} p_{j}=R \sum_{j \notin J_{0}} p_{j}$, then $N_{i}$ will do. Since $R \subset N_{i}$, each $N_{i}$ is of type $\mathrm{II}_{1}$, and if $K_{i} \in \mathscr{J}(M)$ is such that $\left.\delta\right|_{N_{i}}=\operatorname{ad} K_{i}$, then by 4.6 there exists $T_{i} \in K_{\delta}$ (in fact, in ${ }^{\mathrm{co}}\left\{\delta(u) u^{*} \mid u\right.$ unitary element of $\left.N_{i}\right\} \subset \mathrm{K}_{\delta}$ ) such that ad $T_{i}=\operatorname{ad} K_{i}=\left.\delta\right|_{N_{i}}$. Let $T$ be a weak limit point of $\left\{T_{i}\right\}_{i}$. Then ad $T=\delta$ on $\bigcup N_{i}$, so that by the weak continuity of ad $T$ and $\delta, \operatorname{ad} T=\delta$ on $N=\bar{U} N_{i}{ }^{w}$. Since $N$ is of type $\mathrm{II}_{1}$, by 4.4 we have $T \in \mathscr{J}(M)$. 
7.2. Second reduction. It is sufficient to prove the theorem when $N$ is separable and $M$ is countable decomposable.

Indeed, by the preceding reduction we may assume $N$ is separable. Let $\mathscr{U}_{0}$ be a countable subset in the unitary group $\mathscr{U}$ of $N$, dense in $\mathscr{U}$ in the *-strong operator topology. Let $\left\{p_{i}\right\}_{i \in J}$ be an increasing net of countable decomposable projections of $M$ with $p_{i} \uparrow 1$. By the density of $\mathscr{U}_{0}$ in $\mathscr{U}$, it follows that for each $i, \mathrm{~V}\left\{u p_{i} u^{*} \mid u \in \mathscr{U}\right\}=\mathrm{V}\left\{u p_{i} u^{*} \mid u \in \mathscr{U}_{0}\right\}$, so that if we denote this projection by $s_{i}$, then it is countable decomposable (being a supremum of a countable set of countable decomposable projections) and, moreover, $s_{i} \in N^{\prime} \cap M, s_{i} \uparrow 1$. Define $\delta_{i}: N_{s_{i}} \mapsto s_{i} \mathscr{J}(M) s_{i}=\mathscr{J}\left(M_{s_{i}}\right)$ by $\delta_{i}\left(x s_{i}\right)=s_{i} \delta(x) s_{i}$. Since $s_{i} \in N^{\prime} \cap M, \delta_{i}$ are well-defined derivations. If for each $i$ there exists an element $K_{i} \in \mathscr{J}\left(M_{s_{i}}\right)$ such that $\delta_{i}=$ ad $K_{i}$, then by 4.6 there exists $T_{i} \in K_{\delta}$ such that $s_{i} T_{i} s_{i} \in K_{\delta_{i}} \subset s_{i} K_{\delta} s_{i}$ satisfies $\delta_{i}=\operatorname{ad}\left(s_{i} T_{i} s_{i}\right)$. Let $T$ be a weak limit point in $M$ of the net $\left\{T_{i}\right\}_{i}$ $(\subset M)$. Since $\left\{s_{i}\right\}_{i}$ converges strongly to the identity, $T \in K_{\delta}$ and ad $T=\delta$ on $N$. Then 4.4 applies to get $T \in \mathscr{J}(M)$.

7.3. Third reduction. It is sufficient to prove the theorem when $N$ is separable, $M$ is countable decomposable, and $N^{\prime} \cap M$ contains no finite projections of $M$.

Let $p_{0}=\mathrm{V}\left\{e^{\prime} \in N^{\prime} \cap M \mid e^{\prime}\right.$ finite projection of $\left.M\right\}$. Note that in fact $p_{0}=$ $\bigvee\left\{e^{\prime} \in N^{\prime} \cap M \mid e^{\prime}\right.$ projection with $\left.\varphi\left(e^{\prime}\right)<\infty\right\}$. Indeed, this follows immediately by 2.1 , because given any $e \in N^{\prime} \cap M$ and $p \in \mathscr{Z}(M)$ we have $e p \in N^{\prime} \cap M$. Assume now that $\delta(x)=\delta(x) p_{0}, x \in N$. Then $\mathrm{K}_{\delta}=\mathrm{K}_{\delta} p_{0}$. For each unitary element $u \in N$ define on $\mathrm{K}_{\delta}$ the weakly continuous affine transformation $\mathrm{T}_{u}(x)$ $=u x u^{*}+\delta(u) u^{*}$. Then $\mathrm{T}_{u} \mathrm{~T}_{v}=\mathrm{T}_{u v}$, and since $\mathrm{T}_{u}\left(\delta(v) v^{*}\right)=u \delta(v) v^{*} u^{*}+$ $\delta(u) u^{*}=\delta(u v) v^{*} u^{*}$, it follows that $\mathrm{T}_{u}\left(\mathrm{~K}_{\delta}\right) \subset \mathrm{K}_{\delta}$. Consider on $M$ the seminorms $\mathscr{S}=\left\{\varphi\left(x^{*} x e^{\prime}\right)^{1 / 2}\right.$ for $x \in M \mid e^{\prime}$ finite projection in $N^{\prime} \cap M$ with $\varphi\left(e^{\prime}\right)<$ $\infty$. Then the semigroup of transformations $\mathrm{T}_{u}$ on $\mathrm{K}_{\delta}$ is noncontractive, because if $x, y \in \mathrm{K}_{\delta}, x \neq y$, then $\inf _{u} \varphi\left(u(x-y)^{*}(x-y) u^{*} e^{\prime}\right)=\varphi\left((x-y)^{*}(x-y) e^{\prime}\right)$, and if $\varphi\left((x-y)^{*}(x-y) e^{\prime}\right)=0$, then $x-y=(x-y) p_{0}=(x-y)\left(\vee e^{\prime}\right)=0$ (by the faithfulness of $\varphi$ ). Thus by the Ryll-Nardjewski fixed point theorem (see A.3 in [9]) there exists an element $X \in K_{\delta}$ with $\mathrm{T}_{u}(X)=X$ for all unitary elements $u \in N$. But then $u X u^{*}+\delta(u) u^{*}=X$ and thus $\delta(u)=X u-u X$, and by linearity $\delta(x)=X x-x X$ for all $x \in N$. Since $N$ is of type $\mathrm{II}_{1}$, by 4.4 we get $X \in \mathscr{J}(M)$. Similarly, if $\delta(x)=p_{0} \delta(x)$, for any $x \in N$ we obtain that $\delta$ is implemented by an element in $\mathscr{J}(M)$. It follows that there exists $K \in \mathscr{J}(M)$ such that $(\delta-\operatorname{ad} K)(x)=\left(1-p_{0}\right)(\delta-\operatorname{ad} K)(x)\left(1-p_{0}\right)$. Thus, if we define $\delta_{0}: \quad N_{1-p_{0}} \mapsto M_{1-p_{0}}$ by $\delta_{0}\left(x\left(1-p_{0}\right)\right)=(\delta-\operatorname{ad} K)(x)\left(1-p_{0}\right)$, then $\delta_{0}$ is a well-defined derivation taking values into $\left(1-p_{0}\right) \mathscr{J}(M)\left(1-p_{0}\right)=\mathscr{J}\left(M_{1-p_{0}}\right)$. Since $N_{1-p}^{\prime} \cap M_{1-p_{0}}$ contains no finite projections of $M_{1-p_{0}}$ (see the proof of 6.2 , (2)), this shows that in order to prove the theorem for $N$ separable of type $\mathrm{II}_{1}$ and $M$ countable decomposable, we may in addition assume that $N^{\prime} \cap M$ contains no finite projections of $M$. 
7.4. In the rest of this section we may therefore assume $N$ is separable, $M$ is of countable type, and $N^{\prime} \cap M$ contains no finite projections of $M$.

By 6.2 we may construct subalgebras $A \subset R \subset N$ so that $R$ is an approximately finite-dimensional type $\mathrm{II}_{1}$ von Neumann subalgebra of $N, A$ is a maximal abelian von Neumann subalgebra in $N$, and $A^{\prime} \cap M$ contains no finite projections of $M$. Since $R$ is approximately finite-dimensional, there exists an amenable subgroup of unitary elements $\mathscr{U}$ in $R$ such that $\mathscr{U}^{\prime \prime}=R$. Let $K=$ $\int_{\mathscr{U}} \delta(u) u^{*} \mathrm{~d} \mu(u)$, where $\mu$ is an invariant mean on $\mathscr{U}$. Then, like 2.12 , it is easy to see that ad $K$ equals $\delta$ on $\mathscr{U}$ and thus on $R$. By 4.4 it follows that $K \in \mathscr{J}(M)$. Thus, by taking $\delta$ - ad $K$ instead of $\delta$ if necessary, we may suppose $\delta$ vanishes on $R$ and thus on $A \subset R$.

We show that $\delta=0$ on all $N$ follows from the fact that $\left.\delta\right|_{A}=0$, and this will end the proof of Theorem 1.1.

Assume $\delta \neq 0$. Then there exists a unitary element $v \in M$ such that $\delta(v) \neq 0$. Moreover, there exists a finite projection $e \in M_{\varphi, \psi}^{1}$ such that $\varphi\left(e v^{*} \delta(v) e\right) \neq 0$, because otherwise $\varphi\left(v^{*} \delta(v) x\right)=0$ for any linear combination $x$ of projections $e \in M_{\varphi, \psi}^{1}$ and thus, by taking norm limits, for any $x \in M_{\varphi}$, which implies $v^{*} \delta(v)=0$, a contradiction.

Let $q \in N$ be the support of the normal form $N \ni y \mapsto \varphi(y e)$. Then $q e=e$ and thus there are central projections $p_{n}$ in $N$ so that

(*) $\quad p_{n}$ increases to the central support of $g$ in $N$;

$(* *)$ for each $n$ there is a finite number of unitary elements $u_{1}, \ldots, u_{k(n)}$ in $N$ so that

$$
\bigvee_{i} u_{i} p_{n} q u_{i}^{*}=p_{n}
$$

Now from (*) it follows that if $n$ is large enough, then $\left\|e-p_{n} e p_{n}\right\|_{\varphi}$ is small enough to ensure that $\varphi\left(p_{n} e p_{n} v^{*} \delta(v) p_{n} e p_{n}\right) \neq 0$. Since $A$ is maximal abelian in $N, A \supset \mathscr{Z}(N)$, so that $\delta$ vanishes on $\mathscr{Z}(N)$ and thus on all $p_{n}$. Moreover, if $\delta_{n}$ : $N p_{n} \rightarrow \mathscr{J}\left(p_{n} M p_{n}\right)$ is defined by $\delta_{n}\left(x p_{n}\right)=p_{n} \delta(x) p_{n}$, then $\delta_{n}$ vanishes on $A p_{n}$ and the support projection $e_{n}$ of $\sum_{i} u_{i} p_{n} e p_{n} u_{i}^{*}$ (where $u_{i}$ are as in $(* *)$ ) satisfies $\varphi\left(e_{n}\left(v p_{n}\right)^{*} \delta_{n}\left(v p_{n}\right) e_{n}\right) \neq 0 ; N p_{n} \ni y \mapsto \varphi\left(y e_{n}\right)$ is faithful on $N p_{n} ; e_{n}$ is a finite sum of elements in $M_{\varphi, \psi}^{1}$.

Altogether, these considerations show that, by modifying $N, M, \delta, \varphi$, and $\psi$, if necessary we may suppose we are in the following situation:

(i) $M$ is a countable decomposable semifinite von Neumann algebra with a normal semifinite faithful trace $\varphi$ constructed from a normal faithful state $\psi$ on $\mathscr{Z}(M)$ as in 2.1.

(ii) $N \subset M$ is a separable type $\mathrm{II}_{1}$ von Neumann subalgebra and $A \subset N$ is a maximal abelian von Neumann subalgebra of $N$ such that $A^{\prime} \cap M$ has no finite projections of $M$. 
(iii) $\delta: N \rightarrow \mathscr{J}(M)$ is a derivation that vanishes on $A$.

(iv) $v \in N$ is a unitary element and $e \in M$ is a finite projection satisfying the following properties:

(1) There exists a constant $c \geqslant 1$ such that $\psi(q) \leqslant \varphi(e q) \leqslant c \psi(q)$ for any $q \in \mathscr{Z}(M)$.

(2) $\varphi\left(e v^{*} \delta(v) e\right)=1$ (by suitable amplification of $\delta$ with a scalar).

(3) $N \ni y \mapsto \varphi(y e)$ is faithful.

We now prove that for any $n$ there exist a finite projection $e_{n} \in M$ and a unitary element $w_{n} \in N$ such that
(a) $e_{n} \leqslant e, \varphi\left(e-e_{n}\right) \leqslant 2^{-n}$
(b) $\left\|e_{n} w_{n}^{k} e_{n}\right\|<2^{-n}$ for $k \neq 0,|k| \leqslant n$;
(c) $\varphi\left(e_{n} w_{n}^{k} e_{n}\right)=0$ for $k \neq 0$;
(d) $\left|\varphi\left(e_{n} w_{n}^{-p} \delta\left(w_{n}^{p}\right) e_{n}\right)-1\right|<2^{-n}$ if $n \geqslant p>0$; and
(e) $\left|\varphi\left(e_{n} w_{n}^{-s} \delta\left(w_{n}^{p}\right) e_{n}\right)\right|<2^{-n}$ if $p \neq s$ or $p \leqslant 0,|p|,|s| \leqslant n$.

To do this, let $f_{0} \in M$ be a finite projection such that

$$
\begin{aligned}
\left\|\delta(v)\left(1-f_{0}\right)\right\| & <(5 c n)^{-1} 2^{-n-1},\left\|\left(1-f_{0}\right) v^{-1} \delta(v)\right\| \\
& <(5 c n)^{-1} 2^{-n-1},\left\|\delta\left(v^{-1}\right) v\left(1-f_{0}\right)\right\|<(5 c n)^{-1} 2^{-n-1} .
\end{aligned}
$$

By 2.1 there exists a central projection $p \in \mathscr{Z}(M)$ such that $\varphi\left(f_{0} p\right)<\infty$ and $\psi(1-p) \leqslant\left(5 c(\|\delta\|+1) 2^{n+1}\right)^{-2}$ (in all these inequalities $c \geqslant 1$ is the constant appearing in $(i v))$. Set $f=f_{0} p \vee e$. Then by the preceding Corollary 6.4 there exist unitary elements $w_{n} \in N$ and $u_{n} \in A$ and a projection $e_{n} \in M$ such that
$\left(\mathrm{a}^{\prime}\right) \quad e_{n} \leqslant e, \quad \varphi\left(e-e_{n}\right) \leqslant 2^{-n}$
(b') $\left\|f w_{n}^{k} e_{n}\right\| \leqslant(5 c n(\|\delta\|+1)+1)^{-1} 2^{-n-1}$ for $|k| \leqslant 2 n, k \neq 0$;
(c') $\left\|w_{n}-u_{n} v\right\| \leqslant(5 c n(\|\delta\|+1))^{-1} 2^{-n-1}$ and $\varphi\left(e_{n} w_{n}^{k} e_{n}\right)=0$ for $k \neq 0$ 
It follows that if $n \geqslant k>0$, then

(i)

$$
\begin{aligned}
& \left\|\delta\left(w_{n}^{k}\right) e_{n}-w_{n}^{k-1} \delta\left(w_{n}\right) e_{n}\right\|_{\varphi} \\
& =\left\|\sum_{s=0}^{k-2} w_{n}^{s} \delta\left(w_{n}\right) w_{n}^{k-s-1} e_{n}\right\|_{\varphi} \leqslant \sum_{s=0}^{k-2}\left\|\delta\left(w_{n}\right) w_{n}^{k-s-1} e_{n}\right\|_{\varphi} \\
& \leqslant \sum_{s=0}^{k-2}\left\|\delta\left(u_{n} v\right) w_{n}^{k-s-1} e_{n}\right\|_{\varphi}+(k-1)\|\delta\|\|e\|_{\varphi}\left\|w_{n}-u_{n} v\right\| \\
& =\sum_{s=0}^{k-2}\left\|\delta(v) w_{n}^{k-s-1} e_{n}\right\|_{\varphi}+(k-1) c^{1 / 2}\|\delta\|\left\|w_{n}-u_{n} v\right\| \\
& \leqslant \sum_{s=0}^{k-2}\left\|\delta(v) f w_{n}^{k-s-1} e_{n}\right\|_{\varphi}+(k-1)\|\delta\|\left\|(1-p) e_{n}\right\|_{\varphi} \\
& +(k-1) c^{1 / 2}\left\|\delta(v)\left(1-f_{0}\right)\right\|+5^{-1} c^{-1 / 2} 2^{-n-1} \leqslant 2^{-n-1} c^{-1 / 2} ; \\
& \text { (ii) } \quad\left\|\delta\left(w_{n}^{-k}\right) e_{n}\right\|_{\varphi} \leqslant \sum_{s=0}^{k-1}\left\|\left(w_{n}^{-1}\right)^{s} \delta\left(w_{n}^{-1}\right)\left(w_{n}^{-1}\right)^{k-s-1} e_{n}\right\|_{\varphi} \\
& \leqslant \sum_{s=0}^{k-1}\left\|\left(\delta\left(v^{-1}\right) v\right)\left(u_{n} v\right)^{-1}\left(w_{n}^{-1}\right)^{k-s-1} e_{n}\right\|_{\varphi} \\
& +c^{1 / 2} k\|\delta\|\left\|u_{n} v-w_{n}\right\| \\
& \leqslant \sum_{s=0}^{k-1}\left\|\delta\left(v^{-1}\right) v\left(w_{n}^{-1}\right)^{k-s} e_{n}\right\|_{\varphi}+2 k c^{1 / 2}\|\delta\|\left\|u_{n} v-w_{n}\right\| \\
& \leqslant \sum_{s=0}^{k-1}\left\|\delta\left(v^{-1}\right) v f\left(w_{n}^{-1}\right)^{k-s} e_{n}\right\|_{\varphi}+c^{-1 / 2} k(5 n)^{-1} 2^{-n-1} \\
& +k\|\delta\|\left\|(1-p) e_{n}\right\|_{\varphi}+(2 / 5) c^{-1 / 2} 2^{-n-1} \\
& \leqslant\|\delta\| \sum_{s=0}^{k-1}\left\|f\left(w_{n}^{-1}\right)^{k-s} e_{n}\right\|_{\varphi}+(4 / 5) 2^{-n-1} c^{-1 / 2} \leqslant 2^{-n-1} c^{-1 / 2}
\end{aligned}
$$


Thus for $n \geqslant p>0$ we have by (i), ( $\left.\mathrm{c}^{\prime}\right)$, and the equality $\delta\left(u_{n} v\right)=u_{n} \delta(v)$ :

$$
\begin{aligned}
\left|\varphi\left(e_{n} w_{n}^{-p} \delta\left(w_{n}^{p}\right) e_{n}\right)-1\right| \leqslant & \left|\varphi\left(e_{n} w_{n}^{-1} \delta\left(w_{n}\right) e_{n}\right)-1\right|+2^{-n-1} \\
\leqslant & \left|\varphi\left(e_{n} v^{-1} u_{n}^{-1} \delta\left(u_{n} v\right) e_{n}\right)-1\right|+2 c^{1 / 2}\|\delta\|\left\|w_{n}-u_{n} v\right\| \\
& +2^{-n-1} \\
\leqslant & \left|\varphi\left(e v^{-1} \delta(v) e\right)-1\right|+2^{-n}=2^{-n} .
\end{aligned}
$$

If $n \geqslant p>0$ and $s \neq p$, then by (i), ( $\left.c^{\prime}\right)$, and $\left(\mathrm{b}^{\prime}\right)$ we have:

$$
\begin{aligned}
\left|\varphi\left(e_{n} w_{n}^{-s} \delta\left(w_{n}^{p}\right) e_{n}\right)\right| & \leqslant\left|\varphi\left(e_{n} w_{n}^{-s+p-1} \delta\left(w_{n}\right) e_{n}\right)\right|+2^{-n-1} \\
& \leqslant\left|\varphi\left(e_{n} w_{n}^{-s+p-1} u_{n} v v^{-1} \delta(v) e_{n}\right)\right|+5^{-1} 2^{-n-1}+2^{-n-1} \\
& \leqslant\left|\varphi\left(e_{n} w_{n}^{-s+p} v^{-1} \delta(v) e_{n}\right)\right|+2.5^{-1} 2^{-n-1}+2^{-n-1} \\
& \leqslant\left|\varphi\left(e_{n} w_{n}^{-s+p} f v^{-1} \delta(v) e_{n}\right)\right|+4.5^{-1} \cdot 2^{-n-1}+2^{-n-1} \\
& \leqslant\left(5^{-1}+4.5^{-1}+1\right) 2^{-n-1}=2^{-n} .
\end{aligned}
$$

Finally, if $p<0$, then by (ii) and the Cauchy-Schwartz inequality we have for any $s$ :

$$
\left|\varphi\left(e_{n} w_{n}^{-s} \delta\left(w_{n}^{p}\right) e_{n}\right)\right| \leqslant\left\|\delta\left(w_{n}^{p}\right) e_{n}\right\|_{\varphi}\left\|e_{n}\right\|_{\varphi} \leqslant 2^{-n}
$$

This shows that $e_{n}$ and $w_{n}$ as defined before fulfill conditions (a)-(e).

We now define $A_{n} \subset N$ to be the von Neumann algebra generated by $w_{n}$; $p_{n} \in \mathscr{B}\left(\mathrm{L}^{2}(M, \varphi)\right)$ to be the orthogonal projections onto $\overline{A_{n} e_{n}}$; the isometries $u_{n}$ : $\mathrm{L}^{2}(\mathbb{T}, \mu) \mapsto \mathrm{L}^{2}(M, \varphi)$ (where $\mu$ is the normalized Lebesgue measure on the torus T) to be defined by $u_{n}\left(z^{k}\right)=\varphi\left(e_{n}\right)^{-1 / 2} w_{n}^{k} e_{n}$ and the measure preserving isomorphism $\Psi_{n}: \mathrm{L}^{\infty}(T, \mu) \mapsto\left(A_{n}, \varphi\left(e_{n}\right)^{-1} \varphi\left(. e_{n}\right)\right)$ by $\Psi_{n}\left(z^{k}\right)=w_{n}^{k}$. Moreover, we define $\delta_{n}: \mathrm{L}^{\infty}(\mathbb{T}, \mu) \mapsto \mathscr{B}\left(\mathrm{L}^{2}(\mathbb{T}, \mu)\right)$ by $\delta_{n}(f)=u_{n}^{*} \delta\left(\Psi_{n}(f)\right) u_{n}$ for $f \in \mathrm{L}^{\infty}(\mathbb{T}, \mu)$. Since $p_{n}=u_{n} u_{n}^{*} \in A_{n}^{\prime}$, an easy computation shows that all $\delta_{n}$ are derivations and clearly $\left\|\delta_{n}\right\| \leqslant\|\delta\|$.

Let $\omega$ be a free ultrafilter on $\mathbb{N}$ and denote $\Delta: \mathrm{L}^{\infty}(\mathbb{T}, \mu) \mapsto \mathscr{B}\left(\mathrm{L}^{2}(\mathbb{T}, \mu)\right)$ by $\Delta(f)=w-\lim _{n \rightarrow \omega} \delta_{n}(f)$. Then $\Delta$ is also a derivation and $\|\Delta\| \leqslant\|\delta\|$. We show that if $p$ denotes the orthogonal projection onto the Hardy space $\mathrm{H}^{2}(\mathbb{T}, \mu)$ $=\overline{\operatorname{span}}\left\{z^{k} \mid k>0\right\} \subset \mathrm{L}^{2}(\mathbb{T}, \mu)$, then $\Delta=\operatorname{ad} P$ and $\Delta$ is a continuous function from the unit ball of $L^{\infty}(T, \mu)$ with the norm \|\|$_{2}$ into $\mathscr{B}\left(\mathrm{L}^{2}(T, \mu)\right)$ with the uniform norm. To prove the first assertion, note that by (4) $\left\langle\delta_{n}\left(z^{p}\right) 1, z^{s}\right\rangle=$ $\varphi\left(e_{n} w_{n}^{-s} \delta\left(w_{n}^{p}\right) e_{n}\right)$ tend to 1 for $p=s>0$ and to 0 otherwise, so that $\left\langle\Delta\left(z^{p}\right) 1, z^{s}\right\rangle$ is equal to 1 if $p=s>0$ and to 0 otherwise. Since ad $P$ also satisfies these equalities and $\Delta$, ad $P$ are derivations, it follows that $\left\langle\Delta\left(z^{p}\right) z^{k}, z^{s}\right\rangle=$ 
$\left\langle\right.$ ad $\left.P\left(z^{p}\right) z^{k}, z^{s}\right\rangle$ for all $k, p, s \in \mathbb{Z}$, and thus, by linearity and weak continuity of $\Delta$ and ad $P, \Delta=$ ad $P$.

To prove the second assertion (i.e., the continuity result for $\Delta$ ), note first that

(*) given $\beta>0$, there exists $n_{0} \geqslant 1$ and $\alpha>0$ such that for any $n \geqslant n_{0}$ and $a \in A_{n}$, with $\|a\| \leqslant 1$ and $\varphi\left(e_{n} a^{*} a e_{n}\right)<\alpha$ we have $\|\delta(a)\|<\beta$.

Indeed, since $N \ni x \mapsto \varphi(x e)$ is faithful on $N$ by 4.1 , there exists $\alpha^{\prime}>0$ such that if $a \in N,\|a\| \leqslant 1, \varphi\left(e a^{*} a e\right)<\alpha^{\prime}$, then $\|\delta(a)\|<\beta$. Let $n_{0}$ be such that if $n \geqslant n_{0}$, then $\varphi\left(e-e_{n}\right)<\alpha^{\prime} / 2$. If we take $\alpha=\alpha^{\prime} / 2$ and if $\varphi\left(e_{n} a^{*} a e_{n}\right) \leqslant \alpha$, then we get $\varphi\left(e a^{*} a e\right) \leqslant \varphi\left(e-e_{n}\right)\left\|a^{*} a\right\|+\alpha \leqslant \alpha^{\prime} / 2+\alpha^{\prime} / 2=\alpha^{\prime}$, so that $\||\delta(a)| \mid<\beta$.

Now the required continuity assertion on $\Delta$ states that given any $\beta>0$ there exists $\alpha>0$ such that if $f \in \mathrm{L}^{\infty}(\mathbb{T}, \mu),\|f\| \leqslant 1$, and $\|f\|_{2}<\alpha$, then $\|\Delta(f) \xi\|_{2}<\beta$ for any $\xi \in \mathrm{L}^{2}(\mathbb{T}, \mu),\|\xi\|_{2} \leqslant 1$. In fact it is sufficient to check this for $\xi$ Laurent polynomials, $\xi=\sum_{|k| \leqslant m} \alpha_{k} z^{k}$ (with $\sum\left|\alpha_{k}\right|^{2} \leqslant 1$ ). Let $\alpha$ be the one given by (*). Then if $a_{n}=\Psi_{n}(f)$, we have

$$
\begin{aligned}
& \|\Delta(f) \xi\|_{2} \leqslant \limsup _{n}\left\|\delta_{n}(f) \xi\right\|_{2} \\
& =\underset{n}{\lim \sup _{n}}\left\|p_{n} \delta\left(a_{n}\right) p_{n}\left(\sum_{|k| \leqslant m} \alpha_{k} w_{n}^{k}\right) e_{n}\right\|_{\varphi} \varphi\left(e_{n}\right)^{-1 / 2}
\end{aligned}
$$

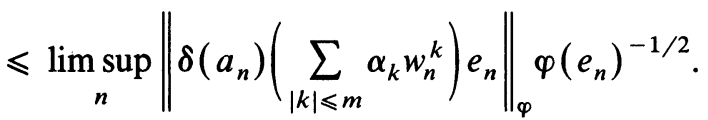

But $\left\|\left(\sum_{|k| \leqslant m} \alpha_{k} w_{n}^{k}\right) e_{n}\right\|^{2}=\left\|\sum_{i, j} \bar{\alpha}_{i} \alpha_{i} e_{n} w_{n}^{j-i} e_{n}\right\| \leqslant \sum_{i}\left|\alpha_{i}\right|^{2}+\sum_{i \neq j}\left|\alpha_{i}\right|\left|\alpha_{j}\right|\left\|e_{n} w_{n}^{j-i} e_{n}\right\|$, and since $\sum_{i, j}\left|\alpha_{i}\right|\left\|\alpha_{j}\right\|=\left(\sum\left|\alpha_{i}\right|\right)^{2} \leqslant(2 m+1) \Sigma\left|\alpha_{i}\right|^{2} \leqslant 2 m+1$, by (b) we get $\left\|\left(\sum \alpha_{k} w_{n}^{k}\right) e_{n}\right\|^{2} \leqslant 1+(2 m+1) 2^{-n}$. Thus, since for $n \geqslant n_{0}$ we have $\left\|\delta\left(a_{n}\right)\right\|<\beta$, it follows that if $n \geqslant n_{0},\left\|\delta\left(a_{n}\right)\left(\sum_{|k| \leqslant m} \alpha_{k} w_{n}^{k}\right) e_{n}\right\|_{\varphi} \leqslant$ $\left(1+(2 m+1) 2^{-n}\right)^{1 / 2} \beta \varphi\left(e_{n}\right)^{1 / 2}$. Hence $\lim _{n} \sup \left\|\delta\left(a_{n}\right)\left(\sum \alpha_{k} w_{n}^{k}\right) e_{n}\right\|_{\varphi} \leqslant \beta \varphi\left(e_{n}\right)^{1 / 2}$ and thus $\|\Delta(f) \xi\|_{2} \leqslant \beta$.

We have thus proved that ad $P$ is continuous from the unit ball of $\mathrm{L}^{\infty}(\mathbb{T}, \mu)$ with the two-norm into $\mathscr{B}\left(\mathrm{L}^{2}(\mathbb{T}, \mu)\right)$ with the uniform norm. But ad $P$ takes values into the finite rank operators for all the polynomials in $\mathrm{L}^{2}(T, \mu)$, so that by the above continuity it follows that ad $P$ takes values into $\mathscr{K}\left(\mathrm{L}^{2}(\mathbb{T}, \mu)\right)$ on all $\mathrm{L}^{\infty}(\mathbb{T}, \mu)$. But then by $\S 5$ (the abelian case of the theorem) ad $P$ is equal to ad $K$ for some $K \in \mathscr{K}\left(\mathrm{L}^{2}(\mathrm{~T}, \mu)\right)$. It follows that $P-K \in \mathrm{L}^{\infty}(\mathrm{T}, \mu)$ and thus $P-K$ is a multiplication operator $M_{f}$ for some function $f \in \mathrm{L}^{\infty}(\mathbb{T}, \mu)\left(\right.$ since $\mathrm{L}^{\infty}(\mathbb{T}, \mu)$ is maximal abelian in $\mathscr{B}\left(\mathrm{L}^{2}(\mathbb{T}, \mu)\right)$. But $1=\lim _{n \rightarrow \infty}\left\langle(P-K) z^{n}, z^{n}\right\rangle=$ $\int z^{-n} f z^{n} \mathrm{~d} \mu(z)=\int f \mathrm{~d} \mu(z)=\lim _{n \rightarrow-\infty}\left\langle(P-K) z^{n}, z^{n}\right\rangle=0$, which is a contradiction.

The initial assumption $\delta \neq 0$ is therefore false and so Theorem 1.1 is completely proved. 
8. The counterexample: Proof of Theorem 1.2. The most simple yet typical situation when the condition of local compatibility between $N$ and $\mathscr{Z}(M)$ is not fulfilled, for abelian (or, more generally, finite type I) $N$ algebras, is when $M$ is the algebra $\mathrm{L}^{\infty}([0,1], \lambda) \otimes \mathscr{B}\left(\mathrm{L}^{2}(\mathbb{T}, \mu)\right)$ and $N=1 \otimes \mathrm{L}^{\infty}(\mathbb{T}, \mu)$, where $\mu$ is the Lebesgue measure on the torus $\mathbb{T}$ and $\lambda$ is the Lebesgue measure on the unit interval $[0,1]$.

It is well known that $\mathrm{L}^{\infty}([0,1], \lambda) \bar{\otimes} \mathscr{B}\left(\mathrm{L}^{2}(\mathbb{T}, \mu)\right)$ can be identified with $\mathbf{L}^{\infty}\left([0,1], \mathscr{B}\left(\mathrm{L}^{2}(\mathbb{T}, \mu)\right)\right.$ with $\mathrm{L}^{\infty}$ having here the obvious significance (i.e., weak $\lambda$-measurable functions of $[0,1]$ into $\mathscr{B}\left(\mathrm{L}^{2}(\mathbb{T}, \mu)\right)$, uniformly bounded, considered modulo a.e. vanishing such functions). Under this identification the ideal $\mathscr{J}(M)$ may be identified with the functions in $\mathrm{L}^{\infty}\left([0,1], \mathscr{B}\left(\mathrm{L}^{2}(\mathrm{~T}, \mu)\right)\right.$ which take values a.e. in $\mathscr{K}\left(\mathrm{L}^{2}(\mathbb{T})\right)$. We denote this set by $\mathrm{L}^{\infty}\left([0,1], \mathscr{K}\left(\mathrm{L}^{2}(\mathbb{T})\right)\right.$. The subalgebra $N=1 \otimes \mathrm{L}^{\infty}(\mathbb{T}, \mu)$ in turn becomes the algebra of all constant, $\mathrm{L}^{\infty}(\mathbb{T}, \mu)$-valued functions on $[0,1]$. Moreover, the center of $M$ may be identified with the scalar-valued functions on $[0,1]$, i.e., $\mathscr{Z}(M)=\mathrm{L}^{\infty}\left([0,1], \mathbb{C} 1_{\mathrm{L}^{2}(\mathbb{T})}\right)$.

Note also that the von Neumann algebra generated by $N$ and $\mathscr{Z}(M)$ is $\tilde{N}=\mathrm{L}^{\infty}\left([0,1], \mathrm{L}^{\infty}(\mathbb{T}, \mu)\right) \subset M=\mathrm{L}^{\infty}\left([0,1], \mathscr{B}\left(\mathrm{L}^{2}(\mathbb{T}, \mu)\right)\right.$ ) (in tensor product terms it equals $L^{\infty}\left([0,1] \bar{\otimes} L^{\infty}(\pi)\right)$.

Now a general observation concerning problems on derivations into $\mathscr{J}(M)$ : By Theorem 1.1, if the von Neumann subalgebra $N$ contains the center of $M$, then any derivation $\delta$ of $N$ into $\mathscr{J}(M)$ is implemented by an element in $\mathscr{J}(M)$; thus, if $N$ does not contain $\mathscr{Z}(M)$, it is natural to try to show that the unique extension of $\delta$ to the von Neumann algebra generated by $N$ and $\mathscr{Z}(M)$ still take values into $\mathscr{J}(M)$. It turns out that this is not always the case. More precisely, we will construct an element $T \in M=\mathrm{L}^{\infty}\left([0,1], \mathscr{B}\left(\mathrm{L}^{2}(\mathbb{T}, \mu)\right)\right)$ so that $[T, N] \subset$ $\mathscr{J}(M)$ but so that $[T, \tilde{N}] \not \subset \mathscr{J}(M)$. Then if $K \in \mathscr{J}(M)$ were such that $T-K \in$ $N^{\prime} \cap M$, it would follow that ad $T=$ ad $K$ on $\tilde{N}$, so that $[T, \tilde{N}] \subset \mathscr{J}(M)$, a contradiction.

The key point of the construction of an element $T$ as above is the following:

8.1. Lemma. There exists $T_{0} \in \mathscr{B}\left(\mathrm{L}^{2}(\mathbb{T}, \mu)\right)$ such that:

(1) Given any measurable set $E \subset \mathbb{T}$ with $1 \in \mathbb{T}$ a point of Lebesgue density 0 or 1 for $E$, the projection $e=\chi_{E} \in \mathrm{L}^{\infty}(\mathbb{T}, \mu)$ satisfies $\left[T_{0}, e\right] \in \mathscr{K}\left(\mathrm{L}^{2}(\mathbb{T}, \mu)\right)$.

(2) There exists a projection $e_{0} \in \mathrm{L}^{\infty}(\mathbb{T}, \mu)$ such that $\left[T_{0}, e_{0}\right] \notin \mathscr{K}\left(\mathrm{L}^{2}(\mathbb{T}, \mu)\right)$.

Before proving this lemma, let us show how one can construct the desired element $T$ in $M$ from the operator $T_{0}$.

8.2. Proposition. Let $U$ be the unitary element in $M=\mathrm{L}^{\infty}([0,1]$, $\left.\mathscr{B}\left(\mathrm{L}^{2}(\mathbb{T}, \mu)\right)\right)$ defined by $U=\left(U_{t}\right)_{0 \leqslant t \leqslant 1}$ with $U_{t}: \mathrm{L}^{2}(\mathbb{T}, \mu) \mapsto \mathrm{L}^{2}(\mathbb{T}, \mu)$, $\left(U_{t} f\right)\left(e^{2 \pi i x}\right)=f\left(e^{2 \pi i(x+t)}\right), x \in[0,1]$. Let $\tilde{T}_{0}$ be the element $T_{0}$ of Lemma 8.1 regarded as a constant function in $M$ (i.e., $\tilde{T}_{0}=1 \otimes T_{0}$ ). Then $T=U \tilde{T}_{0} U^{*}$ satisfies $[T, N] \subset \mathscr{J}(M)$, but there exists no $K \in \mathscr{J}(M)$ so that $\operatorname{ad} T=\operatorname{ad} K$ on $N$.

Proof of 8.2. To prove that $[T, N] \subset \mathscr{J}(M)$ it is sufficient to show that $[T, \tilde{e}] \in \mathscr{J}(M))$ for any projection $\tilde{e} \in N=1 \otimes \mathrm{L}^{\infty}(\mathbb{T}, \mu)$. Thus we have to show 
that given any projection $e \in \mathrm{L}^{\infty}(\mathbb{T}, \mu)$ we have $\left[U_{t} T_{0} U_{t}{ }^{*}, e\right] \in \mathscr{K}\left(\mathrm{L}^{2}(\mathbb{T}, \mu)\right)$ for $\lambda$-almost all $t \in[0,1]$.

Now if $e=\chi_{E}$ for some measurable subset $E \subset \mathbb{T}$, then by Lebesgue's theorem for almost all $t \in[0,1], e^{2 \pi i t}$ has density 0 or 1 . But if $t$ is so that $e^{2 \pi i t} \in \mathbb{T}$ is a point of density 0 or 1 in $E$, then the set $E_{t}$ corresponding to the projection $U_{t}{ }^{*} e U_{t}$ (i.e., $\chi_{E_{t}}=U_{t}{ }^{*} e U_{t}$ ) has density 0 or 1 in the point $1 \in \mathbb{T}$. Thus by $8.1,(1),\left[T_{0}, U_{t}^{*} e U_{t}\right] \in \mathscr{K}\left(\mathrm{L}^{2}(\mathbb{T}, \mu)\right)$, which shows that $\left[U_{t} T_{0} U_{t}{ }^{*}, e\right] \in$ $\mathscr{K}\left(\mathrm{L}^{2}(\mathrm{~T}, \mu)\right)$.

This shows that $\left[U_{t} T_{0} U_{t}{ }^{*}, e\right] \in \mathscr{K} \lambda$-a.e. in $t \in[0,1]$ and proves that $T=U \tilde{T}_{0} U^{*}$ satisfies $[T, N] \subset \mathscr{K}\left(\mathrm{L}^{2}(\mathrm{~T}, \mu)\right)$.

Now if $K \in \mathscr{J}(M)=\mathrm{L}^{\infty}\left([0,1], \mathscr{K}\left(\mathrm{L}^{2}(\mathbb{T}, \mu)\right)\right)$ is such that ad $T=\operatorname{ad} K$ on $N$, then, since the elements in $\mathscr{Z}(M)$ commute with both $T$ and $K$, it follows that ad $T=$ ad $K$ on the von Neumann algebra $\tilde{N}=\mathrm{L}^{\infty}\left([0,1], \mathrm{L}^{\infty}(\mathrm{T}, \mu)\right)$ generated by $N$ and $\mathscr{Z}(M)$. But $U \tilde{N} U^{*}=\tilde{N}$ and more precisely $\tilde{e}=\left(U_{t} e_{0} U_{t}^{*}\right)_{0 \leqslant t \leqslant 1}$ is in $\mathrm{L}^{\infty}\left([0,1], \mathrm{L}^{\infty}(\mathbb{T}, \mu)\right)$, so that $[T, \tilde{e}]=[K, \tilde{e}] \in \mathscr{J}(M)$, which means that $\left[T_{0}, e_{0}\right]=$ $\left[U_{t} T_{0} U_{t}^{*}, U_{t} e_{0} U_{t}^{*}\right] \in \mathscr{K}\left(L^{2}(\mathbb{T}, \mu)\right)$ for $\lambda$-almost all $t \in[0,1]$. But this contradicts $8.1,(2)$.

Q.E.D.

Proof of 8.1. Let $A_{n}, B_{n}$ be subsets of $T$ defined as follows: $A_{n}=$ $\exp \left(2 \pi i\left[1 / 2^{2 n}, 1 / 2^{2 n-1}\right]\right), B_{n}=\exp \left(2 \pi i\left[1 / 2^{2 n+1}, 1 / 2^{2 n}\right]\right)$, for each $n \geqslant 1$.

For an element $f \in \mathrm{L}^{2}(\mathbb{T}, \mu)$ we denote by $\|f\|_{2}$ its norm. We define $\xi_{n}=$ $\left\|\chi_{A_{n}}\right\|_{2}^{-1} \chi_{A_{n}} \in L^{2}(\mathbb{T}, \mu)$ and $\eta_{n}=\left\|\chi_{B_{n}}\right\|_{2}^{-1} \chi_{B_{n}} \in \mathrm{L}^{2}(\mathbb{T}, \mu)$.

Note that $\left\{\xi_{n}\right\}_{n} \cup\left\{\eta_{n}\right\}_{n}$ is an orthonormal family of vectors in $\mathrm{L}^{2}(\mathbb{T}, \mu)$.

If $\xi, \eta \in \mathrm{L}^{2}(\mathbb{T}, \mu)$, we denote by $p_{\xi, \eta}$ the one-dimensional operator in $\mathscr{B}\left(\mathrm{L}^{2}(\mathrm{~T}, \mu)\right)$ defined by

$$
p_{\xi, \eta}(\zeta)=\langle\zeta, \eta\rangle \xi
$$

We define $T_{0}=\sum_{n} p_{\xi_{n}, \eta_{n}}$ (the infinite sum is so-convergent because $\xi_{n}, \eta_{m}$ are all mutually orthogonal vectors). Note that in fact $T_{0}$ is a partial isometry with $T_{0}^{2}=0$.

Let $E \subset \mathbb{T}$ be a measurable set of density zero in 1 . We show that $e=\chi_{E}$ satisfies $e T_{0}, T_{0} e \in \mathscr{K}\left(\mathrm{L}^{2}(\mathbb{T}, \mu)\right)$. Indeed, we have $e T_{0}=\sum p_{\chi_{E} \xi_{n}, \eta_{n}}$. Since the vectors $\left\{\chi_{E} \xi_{n}\right\}_{n}$ are mutually orthogonal in $\mathrm{L}^{2}(T, \mu)$, to show that $e T_{0}$ is compact it is sufficient to show that $\left\|\chi_{E} \xi_{n}\right\|_{2} \mapsto 0$. But

$$
\begin{aligned}
\left\|\chi_{E} \xi_{n}\right\|_{2}^{2} & =\frac{\mu\left(E \cap A_{n}\right)}{\mu\left(A_{n}\right)} \leqslant \frac{\mu\left(E \cap \operatorname { e x p } \left(2 \pi i\left[-1 / 2^{2 n-1}, 1 / 2^{2 n-1}\right]\right.\right.}{\mu\left(A_{n}\right)} \\
& =4 \frac{\mu\left(E \cap \exp \left(2 \pi i\left[-1 / 2^{2 n-1}, 1 / 2^{2 n-1}\right]\right)\right.}{\mu\left(\exp \left(2 \pi i\left[-1 / 2^{2 n-1}, 1 / 2^{2 n-1}\right]\right)\right)},
\end{aligned}
$$

and this last term tends to zero, because 1 is point of density zero for $E$. Similarly, we have $T_{0} e=\sum p_{\xi_{n}, \chi_{E} \eta_{n}} \in \mathscr{K}\left(\mathrm{L}^{2}(\mathrm{~T}, \mu)\right)$. 
Moreover, if $e$ corresponds to a set of density one in 1 , then by the above $\left[T_{0}, 1-e\right] \in \mathscr{K}\left(L^{2}(\mathbb{T}, \mu)\right)$, so that $\left[T_{0}, e\right]$ is also compact.

Now to show that $T_{0}$ also satisfies condition 8.1, (2), let $E_{0} \subset \mathrm{T}$ be a measurable set so that $\mu\left(E_{0} \cap A_{n}\right)=\mu\left(A_{n}\right) / 2$ and $\mu\left(E_{0} \cap B_{n}\right)=\mu\left(B_{n}\right) / 2$ (e.g., take $E_{0}$ to be the union of the halves of each interval $A_{n}$ or $B_{n}$ ).

It is easy to see that if $e_{0}=\chi_{E_{0}}$, then $\left[T_{0}, e_{0}\right]=\sum_{n} p_{\left(1-\chi_{L_{0}}\right)} \xi_{n}, \chi_{L_{0}} \eta_{n}-$ $\sum_{n} p_{\chi_{E_{0}} \xi_{n},\left(1-\chi_{E_{0}}\right) \eta_{n}}$. Moreover, the vectors

$$
\left\{\chi_{E_{0}} \xi_{n},\left(1-\chi_{E_{0}}\right) \xi_{n}, \chi_{E_{0}} \eta_{n},\left(1-\chi_{E_{0}}\right) \eta_{n} \mid n \geqslant 1\right\}
$$

are all mutually orthogonal. Thus, to prove that $\left[T_{0}, e_{0}\right] \notin \mathscr{K}\left(L^{2}(\mathbb{T}, \mu)\right)$ it is sufficient to show that $\left\{\left\|\chi_{E_{0}} \xi_{n}\right\|_{2}^{2}\left\|\left(1-\chi_{E_{0}}\right) \eta_{n}\right\|_{2}^{2}\right\}_{n}$ does not tend to zero. But

$$
\left\|\chi_{E_{0}} \xi_{n}\right\|_{2}^{2}\left\|\left(1-\chi_{E_{0}}\right) \eta_{n}\right\|_{2}^{2}=\frac{\mu\left(E_{0} \cap A_{n}\right)}{\mu\left(A_{n}\right)} \frac{\mu\left(B_{n} \cap E_{0}\right)}{\mu\left(B_{n}\right)}=1 / 4 .
$$

Thus $\left[T_{0}, e_{0}\right] \notin \mathscr{K}\left(\mathrm{L}^{2}(\mathbb{T}, \mu)\right)$, which ends the proof of 8.1.

Q.E.D.

8.3. Final remarks. Theorem 1.2 suggests that in all the cases left uncovered by Theorem 1.1 the derivation problem into the compacts has a negative answer. In fact, with some extra effort one may easily extend the methods of this section to get counterexamples in a large class of cases. However, let us point out here one case left open which deserves attention and for which we could not construct a counterexample:

8.3.1. Problem. Let $M_{0}$ be a type $\mathrm{II}_{1}$ factor, $\mathscr{H}$ an infinite-dimensional Hilbert space and $M=\mathrm{L}^{\infty}([0,1], \lambda) \bar{\otimes} M_{0} \bar{\otimes} \mathscr{B}(\mathscr{H})$. Let $A_{0} \subset M_{0}$ be a (maximal) abelian *-subalgebra of $M_{0}$ and $A_{1} \subset \mathscr{B}(\mathscr{H})$ an atomic (maximal) abelian *-subalgebra of $\mathscr{B}(\mathscr{H})$. Let $N_{0}=1 \otimes A_{0} \otimes 1$ and $N=1 \otimes A_{0} \otimes A_{1}$. Is it true that any derivation of $N_{0}$ (or of $N$ ) into $\mathscr{J}(M)$ is inner?

\section{REFERENCES}

1. R. G. Douglas, Banach Algebra Techniques in Operator Theory, Academic Press, New York and London, 1972.

2. B. E. Johnson, R. V. KAdison, AND J. R. Ringrose, Cohomology of operator algebras, III, Bull. Soc. Math. France 100 (1972), 73-96.

3. B. E. JohnSON AND.S. K. PARROTT, Operators commuting modulo the set of compact operators with a von Neumann algebra, J. Funct. Anal. 11 (1972), 39-61.

4. V. KAFTAL AND G. Weiss, Compact derivations relative to semifinite von Neumann algebras, preprint, 1984.

5. P. I. MuRray AND J. von Neumann, On rings of operators, IV, Ann. of Math. 44 (1943), 716-808.

6. S. PoPA, On a problem of R. V. Kadison on maximal abelian *-subalgebras in factors, Invent. Math. 65 (1981), 269-281.

7. , The commutant modulo the set of compact operators of a von Neumann algebra, J. Funct. Anal. 71 (1987), 393-408. 
8. S. PoPA, Singular maximal abelian *-subalgebras in continuous von Neumann algebras, J. Funct. Anal. 50 (1983), 151-166.

9. S. StRĂTilă AND L. Zsıdo, Lectures on von Neumann Algebras, Editura Academiei, Bucharest and Abacus Press, London, 1979.

10. N. Umegaki, Conditional expectations in an operator algebra, I, Tôhoku Math. J. 6 (1954), 358-362.

11. A. Zygmund, Trigonometric Series, vol. 1, Cambridge University Press, Cambridge, 1959.

Department of Mathematics, National Institute for Scientific and Technical Creation, BD. PĂCII 220, 79622 BUCHAREST, RoMANIA 